\title{
The Van den Bergh duality and the modular symmetry of a Poisson variety
}

\author{
Vasiliy Dolgushev
}

\begin{abstract}
We consider a smooth Poisson affine variety with the trivial canonical bundle over $\mathbb{C}$. For such a variety the deformation quantization algebra $A_{\hbar}$ enjoys the conditions of the Van den Bergh duality theorem and the corresponding dualizing module is determined by an outer automorphism of $A_{\hbar}$ intrinsic to $A_{\hbar}$. We show how this automorphism can be expressed in terms of the modular class of the corresponding Poisson variety. We also prove that the Van den Bergh dualizing module of the deformation quantization algebra $A_{\hbar}$ is free if and only if the corresponding Poisson structure is unimodular.
\end{abstract}

\section{Introduction}

In seminal paper [39] A. Weinstein, inspired by Connes-Takesaki-Tomita theory of modular automorphisms of a von Neumann algebra, introduced a notion of modular class of a smooth real Poisson manifold $M$. This class belongs to the first Poisson cohomology group $H P^{1}(M)$, i.e. the space of Poisson vector fields modulo Hamiltonian vector fields. Independently, J.L. Brylinski and G. Zuckerman [7] introduced and studied the modular vector field in the context of complex analytic Poisson geometry. The modular class is a natural generalization of the modular character of a Lie algebra. This class also admits further generalizations to Lie algebroids [18], to Lie-Rinehart algebras [24], and to $Q$-manifolds [33].

In this paper we show that the deformation quantization incarnation of the modular symmetry of Poisson manifolds is related to the Van den Bergh duality [38] between Hochschild homology and Hochschild cohomology. This relationship can be described in terms of Bursztyn-Waldmann bimodule quantization [8]. More precisely, a modular vector field of a Poisson structure gives us a flat contravariant connection on the $A$-bimodule $A$, where $A$ is the algebra of functions on the Poisson variety. This contravariant connection can be quantized to a bimodule of the deformation quantization algebra $A_{\hbar}$. The main result of the paper (see Theorem 21) states that the resulting bimodule is the Van den Bergh dualizing bimodule $A_{\hbar}$. In this paper we also show that the Van den Bergh dualizing module of $A_{\hbar}$ is isomorphic to $A_{\hbar}$ if and only if the corresponding Poisson structure $\pi$ is unimodular.

The organization of the paper is as follows. In the second section we go over the notation and recall some required results. At the end of this section we describe a construction which produces out of a Poisson vector field a derivation of the deformation quantization algebra. The third section is devoted to the definition of the modular class of a Poisson structure in the algebraic setting. In section 4 we define the modular automorphism 1 of a deformation quantization algebra, formulate our main result (Theorem 21), and prove a useful technical proposition which we need in the proof of Theorem 2. Section 5 is devoted to criterion of unimodularity and section 6 is devoted to the proof of Theorem 2. In the concluding section we discuss some results in literature related to Theorems 2 and 3 . In the Appendix we discuss properties of Poisson, Hamiltonian and log-Hamiltonian vector fields.

\footnotetext{
${ }^{1}$ In [5] K.A. Brown and J.J. Zhang call it the Nakayama automorphism.
} 
Acknowledgment. I would like to thank B. Enriquez, P. Etingof, V. Ginzburg, Y. KosmannSchwarzbach, S. Launois and V. Rubtsov for discussions of this topic. I am grateful to B. Enriquez and V. Rubtsov for their comments about various versions of the manuscript. I am grateful to B. Enriquez for pointing out a significant gap in the original version of the paper. The results of this paper were presented in the seminars at several institutions. These are the University of Angers, University of Montpellier II, ETH in Zürich, and Washington University in St. Louis. I would like to thank the participants of these seminars for their questions and useful comments. This work was in preparation when I was a visitor at FIM at ETH in Zürich. I would like to thank this institute for invitation and for perfect working conditions. I also want to thank my landlord in Zürich Kerry J. Hines-Randle for his hospitality. I am partially supported by the Grant for Support of Scientific Schools NSh-8065.2006.2.

\section{Preliminaries}

For an associative algebra $B$ we denote by $B^{o p}$ the algebra with the opposite multiplication and by $B^{e}$ the enveloping algebra $B^{e}=B \otimes B^{o p}$ of $B$. Der $(B)$ denotes the Lie algebra of derivations of $B . C_{\bullet}(B, N)$ is the Hochschild chain complex of $B$ with coefficients in the $B$-bimodule $N$

$$
C_{\bullet}(B, N)=N \otimes B^{\otimes \bullet}
$$

and $C^{\bullet}(B, N)$ is the Hochschild cochain complex of $B$ with coefficients in $N$

$$
C^{\bullet}(B, N)=\operatorname{Hom}\left(B^{\otimes \bullet}, N\right) .
$$

The Hochschild coboundary operator is denoted by $\partial$ and the Hochschild boundary operator is denoted by $\mathfrak{b}$. Furthermore, we reserve the notation $H H_{\bullet}(B, N)$ for the homology of the complex $\left(C_{\bullet}(B, N), \mathfrak{b}\right)$ and the notation $H H^{\bullet}(B, N)$ for the cohomology of the complex $\left(C^{\bullet}(B, N), \partial\right)$

By convention, $C_{\bullet}(B)$ is the Hochschild chain complex $C \bullet(B, B)$ of $B$ with coefficients in $B$ :

$$
C \bullet(B)=C \bullet(B, B) .
$$

While $C^{\bullet}(B)$ stands for the Hochschild cochain complex of $B$ with coefficients $B$ and with the shifted grading:

$$
C^{\bullet}(B)=C^{\bullet+1}(B, B) .
$$

In particular the lowest component of (2.4) is

$$
C^{-1}(B)=B \text {. }
$$

"DGLA" always means a differential graded Lie algebra. The arrow $\succ \rightarrow$ denotes an $L_{\infty}$-morphism of DGLAs, the arrow $\succ \succ \rightarrow$ denotes a morphism of $L_{\infty}$-modules, and the notation

$$
\begin{aligned}
& L \\
& \downarrow_{\bmod } \\
& M
\end{aligned}
$$

means that $M$ is a DGLA module over the DGLA $L$. The symbol o always stands for the composition of morphisms. $\hbar$ denotes the deformation parameter. 
Throughout this paper $X$ is a smooth complex affine variety of dimension $d$ with the trivial canonical bundle. $A=\mathcal{O}(X)$ denotes the algebra of regular functions on $X$. $T X$ (resp. $T^{*} X$ ) denotes the tangent (resp. cotangent) sheaf of $X$. Since $X$ is smooth, irreducible components of $X$ are exactly its connected components. For this reason it is sufficient to formulate all our proofs in the case of irreducible variety.

We denote by $\Gamma(\mathcal{G})$ the module of global sections of the sheaf $\mathcal{G}$ and by $\Omega^{\bullet}(\mathcal{G})$ the module of exterior forms with values in $\mathcal{G}$. In this paper we only consider quasi-coherent sheaves of modules over $\mathcal{O}_{X}$ and, since $X$ is affine, we tacitly identify all sheaves with the corresponding modules of global sections.

It is well known that the Hochschild cochain complex (2.4) carries the structure of a DGLA. The corresponding Lie bracket (see Eq. (3.2) on page 45 in [12]) was originally introduced by M. Gerstenhaber in [20]. We will denote this bracket by $[,]_{G}$.

The Hochschild chain complex $C \bullet(B)(2.3)$ carries the structure 2 of a DGLA module over the DGLA $C^{\bullet}(B)$. We will denote the action (see Eq. (3.5) on page 46 in [12]) of cochains on chains by $R$.

Due to [23] the cohomology of the complex $C^{\bullet}(A)$ of the algebra $A$ of functions on $X$ is the module $T_{\text {poly }}^{\bullet}(X)$ of polyvector fields on $X$ with the shifted grading

$$
T_{\text {poly }}^{\bullet}(X)=\Gamma\left(\wedge_{\mathcal{O}_{X}}^{\bullet+1} T X\right), \quad T_{\text {poly }}^{-1}(X)=\mathcal{O}(X)
$$

The homology of the complex $C_{\bullet}(A)$ is the module of exterior forms

$$
\mathcal{A}^{\bullet}(X)=\Gamma\left(\wedge_{\mathcal{O}_{X}} T^{*} X\right)
$$

$T_{\text {poly }}^{\bullet}(X)$ is a graded Lie algebra with respect the so-called Schouten-Nijenhuis bracket $[,]_{S N}$ (see Equations $\left.(2.15),(2.16)\right)$ and $\mathcal{A}^{\bullet}(X)$ is a graded Lie algebra module over $T_{\text {poly }}^{\bullet}(X)$ with respect to the Lie derivative $\mathcal{L}(2.13)$. We will regard $T_{\text {poly }}^{\bullet}(X)$ (resp. $\mathcal{A}^{\bullet}(X)$ ) as the DGLA (resp. the DGLA module) with the vanishing differential.

We denote by $x^{i}$ local coordinates on $X$ and by $y^{i}$ fiber coordinates in the tangent bundle $T X$. Having these coordinates $y^{i}$ we can introduce another local basis of exterior forms $\left\{d y^{i}\right\}$. We will use both bases $\left\{d x^{i}\right\}$ and $\left\{d y^{i}\right\}$. In particular, the notation $\Omega^{\bullet}(\mathcal{G})$ is reserved for the module of $d y$-exterior forms with values in the sheaf $\mathcal{G}$ while $\mathcal{A}^{\bullet}(X)(2.6)$ denotes the module of $d x$-exterior forms.

$\mathcal{S} X$ is the formally completed symmetric algebra of the cotangent bundle $T^{*} X$. Elements of the algebra $\mathcal{S} X$ can be viewed as formal power series in tangent coordinates $y^{i}$. We regard $\mathcal{S} X$ as the algebra over $A$. In particular, $C^{\bullet}(\mathcal{S} X)$ (resp. $\left.C_{\bullet}(\mathcal{S} X)\right)$ is the Hochschild cochain (resp. chain) complex of $\mathcal{S} X$ over $A$.

As in [12] the tensor product in

$$
C_{k}(\mathcal{S} X)=\underbrace{\mathcal{S} X \hat{\otimes}_{A} \mathcal{S} X \hat{\otimes}_{A} \ldots \hat{\otimes}_{A} \mathcal{S} X}_{k+1}
$$

is completed in the adic topology in fiber coordinates $y^{i}$ on the tangent bundle $T X$. Similarly, the Hochschild cochains of $\mathcal{S} X$ are substituted by the formal fiberwise polydifferential operators (see Definition 12 on page 60 in [12]).

The cohomology of the complex $C^{\bullet}(\mathcal{S} X)$ is the module $\mathcal{T}_{\text {poly }}^{\bullet}$ of fiberwise polyvector fields (see Definition 11 on page 60 in [12]). The homology of the complex $C \bullet(\mathcal{S} X)$ is the module $\mathcal{E}^{\bullet}$ of fiberwise differential forms (see page 62 in [12]). These are $d x$-forms with values in

\footnotetext{
${ }^{2}$ To be more precise, it is the Hochschild chain complex with the reversed grading $C_{-} \bullet(B)$ which carries this DGLA module structure.
} 
$\mathcal{S} X$. As $T_{\text {poly }}^{\bullet}(X)$, the module $\mathcal{T}_{\text {poly }}^{\bullet}$ is also equipped with a Schouten-Nijenhuis bracket. Similarly $\mathcal{E}^{\bullet}$ forms a graded Lie algebra module over $\mathcal{T}_{\text {poly }}^{\bullet}$. We will use the same notation for the bracket $[,]_{S N}$ and for the Lie derivative $\mathcal{L}$. However, we have to keep in mind that, unlike on $T_{\text {poly }}^{\bullet}(X)$ and $\mathcal{A}^{\bullet}(X)$, the operations $[,]_{S N}$ and $\mathcal{L}$ on $\mathcal{T}_{\text {poly }}^{\bullet}$ and $\mathcal{E}^{\bullet}$ are $A$-linear.

In [12] (see Theorem 4 on page 68) it is shown (in the $C^{\infty}$ setting) that the algebra $\Omega^{\bullet}(\mathcal{S} X)$ can be equipped with a differential of the following form

$$
D=\nabla-\delta+\Lambda
$$

where

$$
\nabla=d y^{i} \frac{\partial}{\partial x^{i}}-d y^{i} \Gamma_{i j}^{k}(x) y^{j} \frac{\partial}{\partial y^{k}}
$$

is a torsion free connection with Christoffel symbols $\Gamma_{i j}^{k}(x)$,

$$
\delta=d y^{i} \frac{\partial}{\partial y^{i}}
$$

and

$$
\Lambda=\sum_{p=2}^{\infty} d y^{k} \Lambda_{k i_{1} \ldots i_{p}}^{j}(x) y^{i_{1}} \ldots y^{i_{p}} \frac{\partial}{\partial y^{j}} \in \Omega^{1}\left(\mathcal{T}_{\text {poly }}^{0}\right) .
$$

We refer to (2.7) as the Fedosov differential.

In our setting it is also possible to construct the Fedosov differential (2.7) since $X$ is affine, and hence $T X$ can be equipped with the desired connection.

Notice that $\delta$ in (2.9) is also a differential on $\Omega^{\bullet}(\mathcal{S} X)$ and (2.7) can be viewed as deformation of $\delta$ via the connection $\nabla$.

According to Proposition 10 on page 64 in [12] the modules $\mathcal{T}_{\text {poly }}^{\bullet}, C^{\bullet}(\mathcal{S} X), \mathcal{E}^{\bullet}$, and $C_{\bullet}(\mathcal{S} X)$ are equipped with the canonical action of the Lie algebra $\mathcal{T}_{\text {poly }}^{0}$ and this action is compatible with the corresponding (DG) algebraic structures. Using this action in chapter 4 of [12] we extend the Fedosov differential (2.7) to differentials on the DGLAs (resp. DGLA modules $) \Omega^{\bullet}\left(\mathcal{T}_{\text {poly }}^{\bullet}\right), \Omega^{\bullet}\left(\mathcal{E}^{\bullet}\right), \Omega^{\bullet}(C \bullet(\mathcal{S} X))$, and $\Omega^{\bullet}\left(C^{\bullet}(\mathcal{S} X)\right)$.

Using acyclicity of the Fedosov differential (2.7) in positive dimension one constructs in [12] embeddings of DGLA modules 3

$$
\begin{array}{ccc}
T_{\text {poly }}^{\bullet}(X) & \stackrel{\lambda_{T}}{\longrightarrow} & \left(\Omega^{\bullet}\left(\mathcal{T}_{\text {poly }}^{\bullet}\right), D,[,]_{S N}\right) \\
\downarrow_{\text {mod }} & \downarrow_{\text {mod }} & \\
\mathcal{A}^{\bullet}(X) & \stackrel{\lambda_{\mathcal{A}}}{\longrightarrow} & \left(\Omega^{\bullet}\left(\mathcal{E}^{\bullet}\right), D\right), \\
\left(\Omega^{\bullet}\left(C^{\bullet}(\mathcal{S} X)\right), D+\partial,[,]_{G}\right) & \stackrel{\lambda_{D}}{\longleftarrow} & C^{\bullet}(A) \\
\downarrow_{\text {mod }} & & \downarrow_{\text {mod }} \\
\left(\Omega^{\bullet}(C \bullet(\mathcal{S} X)), D+\mathfrak{b}\right) & \stackrel{\lambda_{C}}{\longleftarrow} & C \bullet(A),
\end{array}
$$

and shows that these are quasi-isomorphisms of the corresponding complexes.

\footnotetext{
${ }^{3}$ See Eq. (5.1) on page 81 in [12].
} 
Furthermore, using Kontsevich's and Shoikhet's formality theorems for $\mathbb{R}^{d}[27]$, [35] in [12] one constructs the following commutative diagram

$$
\begin{array}{ccc}
\left(\Omega^{\bullet}\left(\mathcal{T}_{\text {poly }}^{\bullet}\right), D,[,]_{S N}\right) & \succ \mathcal{K} & \left(\Omega^{\bullet}\left(C^{\bullet}(\mathcal{S} X)\right), D+\partial,[,]_{G}\right) \\
\downarrow & \downarrow \\
\downarrow_{\text {mod }} & & \downarrow_{\text {mod }} \\
\left(\Omega^{\bullet}\left(\mathcal{E}^{\bullet}\right), D\right) & \leftarrow \mathcal{S} \prec & \left(\Omega^{\bullet}(C \bullet(\mathcal{S} X)), D+\mathfrak{b}\right)
\end{array}
$$

where $\mathcal{K}$ is an $L_{\infty}$ quasi-isomorphism of DGLAs, $\mathcal{S}$ is a quasi-isomorphism of $L_{\infty}$-modules over the DGLA $\left(\Omega^{\bullet}\left(\mathcal{T}_{\text {poly }}^{\bullet}\right), D,[,]_{S N}\right)$, and the $L_{\infty}$-module structure on $\Omega^{\bullet}(C \bullet(\mathcal{S} X))$ is obtained by composing the quasi-isomorphism $\mathcal{K}$ with the DGLA modules structure $R$ (see Eq. (3.5) on p. 46 in [12] for the definition of $R$ ).

Remark. As in [12] we use adapted versions of Hochschild (co)chains for the algebra of functions $A$. Thus, $C^{\bullet}(A)$ is the complex of (algebraic) polydifferential operators (see page 48 in [12]) on $X$ and $C \cdot(A)$ is the sheaf of polyjets as in Equation (3.15) on page 49 in [12]. In the algebraic setting these complexes are quasi-isomorphic to the corresponding genuine Hochschild complexes (2.3) and (2.4) of $A$.

Let us recall the formulas of Cartan calculus of exterior forms and polyvector fields.

The Lie derivative with respect to a polyvector field $\gamma$ is defined as the graded commutator

$$
\mathcal{L}_{\gamma}=\left[d, i_{\gamma}\right]
$$

of the de Rham differential $d$ and the contraction $i_{\gamma}$ with $\gamma$. The contraction is defined in the obvious way for $\gamma$ being a function or a vector and then extended to an arbitrary polyvector by the equation

$$
i_{\gamma_{1}} i_{\gamma_{2}}=i_{\gamma_{1} \wedge \gamma_{2}}, \quad \gamma_{1}, \gamma_{2} \in T_{\text {poly }}^{\bullet}(X) .
$$

The Schouten-Nijenhuis bracket $[,]_{S N}$ on $T_{\text {poly }}^{\bullet}(X)$ is defined by the equations

$$
\begin{gathered}
{[a, b]_{S N}=0, \quad[w, a]_{S N}=w(a), \quad\left[w_{1}, w_{2}\right]_{S N}=\left[w_{1}, w_{2}\right]} \\
{\left[\gamma_{1}, \gamma_{2} \wedge \gamma_{3}\right]_{S N}=\left[\gamma_{1}, \gamma_{2}\right]_{S N} \wedge \gamma_{3}+(-1)^{\left|\gamma_{1}\right|\left(\left|\gamma_{2}\right|+1\right)} \gamma_{2} \wedge\left[\gamma_{1}, \gamma_{3}\right]_{S N}, \quad \gamma_{i} \in T_{\text {poly }}^{\bullet}(X) .}
\end{gathered}
$$

where $a, b$ are functions, $w, w_{1}, w_{2}$ are vector fields, [, ] stands for the Lie bracket of vector fields, and $\left|\gamma_{i}\right|$ denotes the degree of the polyvector $\gamma_{i}$ in $T_{\text {poly }}^{\bullet}(X)(2.5)$.

The Lie derivative and the contraction operation satisfy the following equations

$$
\begin{gathered}
{\left[i_{\gamma_{1}},\left[d, i_{\gamma_{2}}\right]\right]=i_{\left[\gamma_{1}, \gamma_{2}\right]_{S N}},} \\
{\left[\mathcal{L}_{\gamma_{1}}, \mathcal{L}_{\gamma_{2}}\right]=\mathcal{L}_{\left[\gamma_{1}, \gamma_{2}\right]_{S N}} .}
\end{gathered}
$$

Let us also recall the Van den Bergh duality theorem:

Theorem 1 (M. Van den Bergh, [38]) If $B$ is a finitely generated bimodule coherent algebra of finite Hochschild dimension d,

$$
\operatorname{Ext}_{B^{e}}^{m}(B, B \otimes B)= \begin{cases}U_{B} & \text { if } m=d, \\ 0 & \text { otherwise }\end{cases}
$$

and $U_{B}$ is an invertible 5 -bimodule then for every B-bimodule $N$

$$
H H^{\bullet}(B, N) \cong H H_{d-\bullet}\left(B, U_{B} \otimes_{B} N\right) .
$$

\footnotetext{
${ }^{4} \mathrm{An}$ algebra is called bimodule coherent if every map between finite rank free $B$-bimodules has a finitely generated kernel (see Definition 3.5.1 in [21).

${ }^{5} \mathrm{~A} B$-bimodule $U$ is called invertible if there is a $B$-bimodule $V$ such that $U \otimes_{B} V \cong B$.
} 
In Equation (2.19) $B \otimes B$ is considered as a bimodule over $B$ with respect to the external $B$ bimodule structure. It is the internal $B$-bimodule structure which equips all the Ext groups $\operatorname{Ext}_{B^{e}}(B, B \otimes B)$ with a structure of $B$-bimodule.

We refer to $U_{B}$ as the Van den Bergh dualizing module of $B$.

\subsection{Quantization of the derivations of Poisson algebra}

Let $\pi_{1}$ be a Poisson structure on $X$ and $A_{\hbar}=(A[[\hbar]], *)$ be a deformation quantization of $\pi_{1}$ in the sense of [2] and [3]. Let, furthermore,

$$
\pi=\hbar \pi_{1}+\hbar^{2} \pi_{2}+\cdots \in \hbar \Gamma\left(\wedge^{2} T X\right)[[\hbar]]
$$

be a representative of Kontsevich's class of the deformation $A_{\hbar}$.

Due to the Jacobi identity $[\pi, \pi]_{S N}=0$ we get a non-zero differential

$$
\partial_{\pi}=[\pi,]_{S N}
$$

on $T_{\text {poly }}^{\bullet}(X)[[\hbar]]$. This differential was originally introduced by Lichnerowicz in [32] and the cohomology of the complex

$$
\left(T_{\text {poly }}^{\bullet}(X)[[\hbar]],[\pi,]_{S N}\right)
$$

is the desuspended Poisson cohomology of $\pi$ :

$$
H P^{\bullet}(X, \pi)=H^{\bullet-1}\left(T_{\text {poly }}^{\bullet}(X)[[\hbar]],[\pi,]_{S N}\right) .
$$

In particular, the zeroth Poisson cohomology is exactly the $\mathbb{C}[[\hbar]]$-module of Casimir functions of $\pi$ and the first Poisson cohomology is the quotient of Poisson vector fields of $\pi$ by Hamiltonian vector fields.

In this subsection we construct a $\mathbb{C}[[\hbar]]$-linear map

$$
w \rightarrow \mathcal{D}_{w}: \Gamma(T X)[[\hbar]] \cap \operatorname{ker}[\pi,]_{S N} \rightarrow \operatorname{Der}\left(A_{\hbar}\right)
$$

satisfying the following properties:

$$
\begin{gathered}
\mathcal{D}_{w}=w \quad \bmod \hbar \\
{\left[\mathcal{D}_{w_{1}}, \mathcal{D}_{w_{2}}\right]=\mathcal{D}_{\left[w_{1}, w_{2}\right]}+\text { inner derivations }} \\
\forall \quad w, w_{1}, w_{2} \in \Gamma(T X)[[\hbar]] \cap \operatorname{ker}[\pi,]_{S N} .
\end{gathered}
$$

First, the Poisson structure $\pi(2.20)$ lifts to a Maurer-Cartan element $\lambda_{T}(\pi)$ in $\Omega^{\bullet}\left(\mathcal{T}_{\text {poly }}^{\bullet}\right)[[\hbar]]$ which is flat with respect to the Fedosov differential $D$ (2.7). Using this element we extend the differential $D$ on $\Omega^{\bullet}\left(\mathcal{T}_{\text {poly }}^{\bullet}\right)[[\hbar]]$ to

$$
D+\left[\lambda_{T}(\pi),\right]_{S N}: \Omega^{\bullet}\left(\mathcal{T}_{\text {poly }}^{\bullet}\right)[[\hbar]] \rightarrow\left(\Omega^{\bullet}\left(\mathcal{T}_{\text {poly }}^{\bullet}\right)[[\hbar]]\right)[1],
$$

where [1] denotes the shift of the total degree by 1 .

Second, the star-product $*$ viewed as an element in $C^{1}(A)[[\hbar]]$ lifts to a $D$-flat cochain in $C^{1}(\mathcal{S} X)[[\hbar]]$ and hence gives us a new associative product on $\mathcal{S} X[[\hbar]]$

$$
\diamond=\lambda_{D}(*)
$$

compatible with the differential $D$ (2.7) .

Using this product we extend the original differential $D+\partial$ on $\Omega^{\bullet}\left(C^{\bullet}(\mathcal{S} X)\right)[[\hbar]]$ to

$$
D+\partial_{\diamond}: \Omega^{\bullet}\left(C^{\bullet}(\mathcal{S} X)\right)[[\hbar]] \rightarrow\left(\Omega^{\bullet}\left(C^{\bullet}(\mathcal{S} X)\right)[[\hbar]]\right)[1]
$$


where $\partial_{\diamond}$ is the Hochschild coboundary operator corresponding to the new product $\diamond$ on $\mathcal{S} X[[\hbar]]$ and $[1]$ as above denotes the shift of the total degree by 1 .

Next, following the lines of section 5.3 in [12] we can construct the following chain of $\left(L_{\infty}\right)$ quasi-isomorphisms of DGLAs

$$
\left(T_{\text {poly }}^{\bullet}(X)[[\hbar]],[\pi,]_{S N}\right) \stackrel{\lambda_{T}}{\longrightarrow} \Omega^{\bullet}\left(\mathcal{T}_{\text {poly }}^{\bullet}\right)[[\hbar]] \stackrel{\widetilde{\mathcal{K}}}{\rightarrow} \Omega^{\bullet}\left(C^{\bullet}(\mathcal{S} X)\right)[[\hbar]] \stackrel{\lambda_{D}}{\longleftarrow} C^{\bullet}\left(A_{\hbar}\right),
$$

where $\Omega^{\bullet}\left(\mathcal{T}_{\text {poly }}^{\bullet}\right)[[\hbar]]$ (resp. $\left.\Omega^{\bullet}\left(C^{\bullet}(\mathcal{S} X)\right)[[\hbar]]\right)$ carries the differential (2.26) (resp. the differential (2.28) $)$.

The maps $\lambda_{T}$ (2.10) and $\lambda_{D}$ (2.11) are genuine morphisms of DGLAs and $\widetilde{\mathcal{K}}$ is an $L_{\infty}$-quasiisomorphism obtained from $\mathcal{K}$ in $(2.12)$ by twisting via the Maurer-Cartan element $\lambda_{T}(\pi)$ and adjusting by the action of the prounipotent group corresponding to the Lie algebra

$$
\mathfrak{g}=\hbar C^{0}(\mathcal{S} X)[[\hbar]] \oplus \hbar \Omega^{1}\left(C^{-1}(\mathcal{S} X)\right)[[\hbar]] .
$$

Let $w \in \Gamma(T X)[[\hbar]]$ be a Poisson vector field of $\pi$. In other words, $w$ is a degree zero cocycle in the complex $T_{\text {poly }}^{\bullet}(X)[[\hbar]]$ with the differential $[\pi,]_{S N}$. The map $\lambda_{T}$ in the chain (2.29) pulls $w$ to a degree zero cocycle $\lambda_{T}(w)$ in the complex $\Omega^{\bullet}\left(\mathcal{T}_{\text {poly }}^{\bullet}\right)[[\hbar]]$ with the differential $(2.26)$. Then, using the structure map $\widetilde{\mathcal{K}}_{1}$ of the first level of the $L_{\infty}$-morphism $\widetilde{\mathcal{K}}$, we get a cocycle

$$
W=\widetilde{\mathcal{K}}_{1}\left(\lambda_{T}(w)\right)
$$

in the complex $\Omega^{\bullet}\left(C^{\bullet}(\mathcal{S} X)\right)[[\hbar]]$ with the differential (2.28) .

As a cocycle of degree zero, $W$ has two components:

$$
W=W_{0}+W_{1}, \quad W_{0} \in \Omega^{0}\left(C^{0}(\mathcal{S} X)\right)[[\hbar]], \quad W_{1} \in \Omega^{1}(\mathcal{S} X)[[\hbar]] .
$$

Recall that, because of the shift (2.4),$C^{0}(\mathcal{S} X)=C^{1}(\mathcal{S} X, \mathcal{S} X)$ and $C^{-1}(\mathcal{S} X)=\mathcal{S} X$.

Since the Fedosov differential (2.7) is acyclic in positive exterior degree we can kill the component $W_{1}$ by adding an exact term to $W$. Namely, using the homotopy operator $\Phi$ (see Eq. (4.36) in chapter 4 of [12]) for the Fedosov differential, we conclude that the cocycle

$$
U=W_{0}-\partial_{\diamond} \Phi\left(W_{1}\right) \in \Omega^{0}\left(C^{0}(\mathcal{S} X)\right)[[\hbar]]
$$

is cohomologous to $W$. If we denote by $\operatorname{pr}_{1}$ the projection

$$
\operatorname{pr}_{1}: \Omega^{0}\left(C^{0}(\mathcal{S} X)\right)[[\hbar]] \oplus \Omega^{1}(\mathcal{S} X)[[\hbar]] \rightarrow \Omega^{1}(\mathcal{S} X)[[\hbar]]
$$

onto the degree 1 exterior forms then $U$ can be rewritten as

$$
U=W-\left(D+\partial_{\diamond}\right) \Phi\left(\operatorname{pr}_{1}(W)\right) .
$$

Since $U$ has the only non-zero component in the exterior degree 0 the equation $\left(D+\partial_{\diamond}\right) U=0$ is equivalent to the pair of equations

$$
D U=0, \quad \partial_{\diamond} U=0 .
$$

On the other hand, by definition, the map $\lambda_{D}$ in $(2.29)$ identifies $C^{\bullet}\left(A_{\hbar}\right)$ with $C^{\bullet}(\mathcal{S} X) \cap$ ker $D$. Hence, the equation

$$
\lambda_{D}\left(\mathcal{D}_{w}\right)=W-\left(D+\partial_{\diamond}\right) \Phi\left(\operatorname{pr}_{1}(W)\right)
$$

defines an element $\mathcal{D}_{w}$ in $C^{1}\left(A_{\hbar}\right)$. Furthermore, since $\partial_{\diamond} U=0$ the element $\mathcal{D}_{w}$ is a derivation of $A_{\hbar}$. We take (2.30) and (2.33) as the equations defining the desired map $\mathcal{D}(2.23)$. 
Equation (2.24) follows from the construction while Equation (2.25) follows from the identity between structure maps $\widetilde{\mathcal{K}}_{1}$ and $\widetilde{\mathcal{K}}_{2}$ of $\widetilde{\mathcal{K}}$ :

$$
\begin{gathered}
{\left[\widetilde{\mathcal{K}}_{1}\left(\gamma_{1}\right), \widetilde{\mathcal{K}}_{1}\left(\gamma_{2}\right)\right]_{G}-\widetilde{\mathcal{K}}_{1}\left(\left[\gamma_{1}, \gamma_{2}\right]_{S N}\right)=\left(D+\partial_{\diamond}\right) \widetilde{\mathcal{K}}_{2}\left(\gamma_{1}, \gamma_{2}\right)+\widetilde{\mathcal{K}}_{2}\left(D \gamma_{1}, \gamma_{2}\right)+(-1)^{\left|\gamma_{1}\right|} \widetilde{\mathcal{K}}_{2}\left(\gamma_{1}, D \gamma_{2}\right)+} \\
\widetilde{\mathcal{K}}_{2}\left(\left[\lambda_{T}(\pi), \gamma_{1}\right]_{S N}, \gamma_{2}\right)+(-1)^{\left|\gamma_{1}\right|} \widetilde{\mathcal{K}}_{2}\left(\gamma_{1},\left[\lambda_{T}(\pi), \gamma_{2}\right]_{S N}\right) \\
\gamma_{1}, \gamma_{2} \in \Omega^{\bullet}\left(\mathcal{T}_{\text {poly }}^{\bullet}\right)[[\hbar]]
\end{gathered}
$$

where $\left|\gamma_{1}\right|$ is the degree of $\gamma_{1}$.

\section{Classical modular symmetry}

Let $X$ be a smooth affine variety over $\mathbb{C}$ with a trivial canonical class and with $\pi_{1} \in \Gamma\left(\wedge^{2} T X\right)$ being a Poisson structure.

Following M. Kontsevich [27] equivalence classes of star-products of $\pi_{1}$ on $X$ can be parameterized using deformed Poisson structures. Those are formal power series in $\hbar$ of sections of $\wedge^{2} T X$

$$
\pi=\hbar \pi_{1}+\hbar^{2} \pi_{2}+\ldots
$$

starting with $\hbar \pi_{1}$ and satisfying the Jacobi identity:

$$
[\pi, \pi]_{S N}=0 .
$$

This motivates us to replace polyvector fields and exterior forms by the corresponding formal power series in $\hbar$. In particular, it makes sense to consider the Poisson structure (3.1), as well as its Poisson and Hamiltonian vector fields from the very beginning.

In this section we define the modular class [7], [39] of the Poisson structure $\pi$ (3.1) on a smooth affine variety $X$ with trivial canonical bundle. Unlike in the real $C^{\infty}$ setting as in [39], or complex analytic setting as in [7], in our case it is no longer possible to take log of nowhere vanishing functions. This is why the modular class is not a class in the first Poisson cohomology of $\pi$. Instead it is defined by a Poisson vector field considered modulo the so-called log-Hamiltonian vector fields. We introduce and discuss the latter fields in the Appendix.

Let $\omega$ be a nowhere vanishing regular section of the canonical bundle $\wedge^{d} T^{*} X$ on $X$. Since the bundle $\wedge^{d} T^{*} X$ is trivial such a section always exists and it is defined up to a multiplication by a unit $f$ of $A=\mathcal{O}_{X}$ :

$$
\omega \rightarrow f \omega
$$

Let us define the modular vector field $v \in \hbar \Gamma(T X)[[\hbar]]$ of the Poisson structure $\pi$ (3.1) by the equation:

$$
i_{v} \omega=\mathcal{L}_{\pi} \omega
$$

The vector field $v$ is well defined since $\omega$ is nowhere vanishing.

Let us show that $v$ is a Poisson vector field of $\pi$ or in other words,

$$
[\pi, v]_{S N}=0 .
$$

Indeed, using (2.17), (2.18), (3.2), and (3.4) we get

$$
i_{[\pi, v]_{S N}} \omega=-i_{[v, \pi]_{S N}} \omega=-i_{v} \mathcal{L}_{\pi} \omega-\mathcal{L}_{\pi} i_{v} \omega=-i_{v} i_{v} \omega-\mathcal{L}_{\pi} \mathcal{L}_{\pi} \omega=0 .
$$


Therefore, since $\omega$ is nowhere vanishing, the bivector field $[\pi, v]_{S N}$ should vanish.

If we alter the section $\omega$ according to (3.3) the field $v$ differs as

$$
v \rightarrow v-f^{-1}[\pi, f]_{S N} .
$$

Thus, we see that $v$ is defined up to adding the log-Hamiltonian vector fields (See the Appendix.).

Remark. We might as well use the formal power series of top degree forms in $\hbar$

$$
\widetilde{\omega}=\omega_{0}+\hbar \omega_{1}+\hbar^{2} \omega_{2}+\ldots
$$

starting with a nowhere vanishing form $\omega_{0}$ instead of $\omega$ in the definition of the modular vector field (3.4).

Following [39] we say that

Definition 1 A Poisson structure $\pi$ (3.1) is called unimodular if its modular class is trivial.

As we are working in the algebraic setting the triviality of the modular class means that a modular vector field of $\pi$ is a log-Hamiltonian vector field of $\pi$. In other words, there exists a unit $f$ in $A[[\hbar]]$ such that

$$
\mathcal{L}_{\pi} \omega-f^{-1} i_{[\pi, f]_{S N}} \omega=0
$$

where $\omega$ is the nowhere vanishing top degree form which was used to define the modular vector field.

Using the formulas of Cartan calculus (2.13), (2.17), and (2.18) it is not hard to show that Equation (3.8) is equivalent to

$$
\mathcal{L}_{\pi}(f \omega)=0 .
$$

Thus, if $\pi$ is unimodular then there exists a formal power series $\widetilde{\omega}$ (3.7) of top degree forms satisfying the equation

$$
\mathcal{L}_{\pi} \widetilde{\omega}=0 .
$$

Adding to this observation the obvious reverse statement we get

Proposition 1 The Poisson structure $\pi$ (3.1) is unimodular if and only if there exits a formal power series of top degree forms

$$
\widetilde{\omega}=\omega_{0}+\hbar \omega_{1}+\cdots \in \Omega^{d}(X)[[\hbar]]
$$

starting with a nowhere vanishing form $\omega_{0}$ such that

$$
\mathcal{L}_{\pi} \widetilde{\omega}=0 .
$$

Examples. If the Poisson variety $\left(X, \pi_{1}\right)$ is symplectic then any Poisson structure $\pi$ of the form (3.1) is unimodular. Indeed, in this case one can produce the inverse $\eta$ for the series of bivectors

$$
\pi_{1}+\hbar \pi_{2}+\hbar^{2} \pi_{3}+\ldots
$$

Due to (3.2) $\eta$ is a formal series in $\hbar$ of closed two forms starting with $\pi_{1}^{-1}$ and $\wedge^{d / 2} \eta$ is the desired volume form (3.7) satisfying Equation (3.10). An example of a Poisson structure with a non-trivial modular class is provided by the dual space $\mathfrak{h}^{*}$ of a finite dimensional Lie algebra $\mathfrak{h}$ with a non-zero modular character. 


\section{Quantum modular symmetry}

Let $A_{\hbar}=(A[[\hbar]], *)$ be a deformation quantization algebra of the Poisson variety $\left(X, \pi_{1}\right)$ in the sense of [2] and [3]. We regard $A_{\hbar}$ as an algebra over the ring $\mathbb{C}[[\hbar]]$.

Proposition 2 The algebra $A_{\hbar}$ has the Hochschild dimension $d=\operatorname{dim} X$. It satisfies the conditions of Theorem 1 . The dualizing bimodule of $A_{\hbar}$ is isomorphic to $A_{\hbar} \nu$, where $\nu$ is an automorphism of $A_{\hbar}$ satisfying the property

$$
\nu=I d \quad \bmod \hbar .
$$

Proof. Using the standard arguments of deformation theory (see Proposition 6 in [13] or step 2 in the proof of Theorem 4.1 in [17]) one can show that the algebra $A_{\hbar}$ (over $\left.\mathbb{C}[[\hbar]]\right)$ has the same Hochschild dimension as the algebra $A$,

$$
\operatorname{Ext}_{A_{\hbar}^{e}}^{k}\left(A_{\hbar}, A_{\hbar} \otimes A_{\hbar}\right)=0
$$

for every $k \neq d$, and there is an automorphism $\nu$ of $A_{\hbar}$ such that

$$
\nu=I d \quad \bmod \hbar,
$$

and

$$
\operatorname{Ext}_{A_{\hbar}^{e}}^{d}\left(A_{\hbar}, A_{\hbar} \otimes A_{\hbar}\right) \cong A_{\hbar} \nu
$$

as bimodules over $A_{\hbar}$. Here $A_{\hbar} \otimes A_{\hbar}$ is considered as a bimodule over $A_{\hbar}$ with respect to the external $A_{\hbar}$-bimodule structure. It is the internal $A_{\hbar}$-bimodule structure which equips all the Ext groups $\operatorname{Ext}_{A_{\hbar}^{e}}\left(A_{\hbar}, A_{\hbar} \otimes A_{\hbar}\right)$ with a structure of $A_{\hbar}$-bimodule.

$A_{\hbar}$ is finitely generated since so is $A$. Furthermore, it is not hard to show that bimodule coherence is stable under deformations we consider and the bimodule coherence of the commutative algebra $A$ follows from the fact that $A \otimes_{\mathbb{C}} A$ is Noetherian.

The proposition is proved.

Notice that, $\nu$ is defined up to a composition with an inner automorphism.

Definition 2 We call the outer automorphism $\nu$ the modular automorphism of $A_{\hbar}$.

This definition is motivated by the main result of this paper which relates the outer automorphism $\nu$ of the deformation quantization algebra $A_{\hbar}$ to the modular class of the Poisson structure $\pi$ (2.20). This result can be formulated as follows:

Theorem 2 Let $A_{\hbar}$ be a deformation quantization algebra of $\left(X, \pi_{1}\right)$ and let $\pi$ (2.20) be a representative of Kontsevich's class of $A_{\hbar}$. If $v$ is a modular vector field of the Poisson structure $\pi$ and $\mathcal{D}_{v}$ is a derivation of $A_{\hbar}$ constructed from $v$ via Kontsevich's formality theorem then the modular automorphism $\nu$ of $A_{\hbar}$ is equal to $\exp \left(\mathcal{D}_{v}\right)$ up to an inner automorphism.

Remark. It is Corollary 1 at the end of the Appendix which implies that the automorphism $\exp \left(\mathcal{D}_{v}\right)$ in the above theorem is well defined up to an inner automorphism.

In paper [8] H. Bursztyn and S. Waldmann showed that the semiclassical limit of an invertible bimodule over a deformation quantization algebra is a flat contravariant connection on an invertible bimodule of the corresponding Poisson algebra. They proved that, under certain conditions, such a contravariant connection on an invertible bimodule over $A$ can be quantized to an invertible bimodule over $A_{\hbar}$. On the other hand every Poisson vector field defines a flat contravariant connection of the $A$-bimodule $A$ and two such connections are 
isomorphic if and only if the corresponding Poisson vector fields differ by a log-Hamiltonian vector field (see Lemma 4.8 in [8]). In terms of [8] Theorem 2 can formulated 6 as follows: the deformation quantization of the contravariant connection corresponding to the modular vector field of a Poisson structure is the Van den Bergh dualizing bimodule of the deformation quantization algebra.

The proof of Theorem 2 is given in section 6. In the remainder of this section we would like to prove an important technical result which we will need later on.

Let $w \in \hbar \Gamma(T X)[[\hbar]]$ be a Poisson vector field of $\pi(2.20)$. Using the map $\mathcal{D}(2.23)$ we produce a derivation $\mathcal{D}_{w}$ of the algebra $A_{\hbar}$. Since the map $\mathcal{D}$ is $\mathbb{C}[[\hbar]]$-linear $\mathcal{D}_{w}=0 \bmod \hbar$. Hence, it makes sense to speak about the following automorphism

$$
\phi_{w}=\exp \left(\mathcal{D}_{w}\right)
$$

of $A_{\hbar}$.

Using this automorphism we define an associative product $\cdot$ on $A_{\hbar}\left[t, t^{-1}\right]$ by the following rule:

$$
\left(a t^{n}\right) \cdot\left(b t^{m}\right)=a * \phi_{w}^{n}(b) t^{n+m},
$$

where $a, b \in A_{\hbar}$ and $n, m$ are arbitrary integers.

This product can be easily extended to a star-product on $A\left[t, t^{-1}\right][[\hbar]]$. In this way we obtain a deformation quantization of the affine variety $X \times \mathbb{C}^{\times}$with the following Poisson structure:

$$
\pi_{1}^{w}=\pi_{1}+t \partial_{t} \wedge w_{1}
$$

where $\pi_{1}$ (resp. $w_{1}$ ) is the first coefficient in the expansion of $\pi$ (2.20) (resp. expansion of $w)$ in $\hbar$ and $\mathbb{C}^{\times}=\mathbb{C} \backslash\{0\}$.

The algebra $A_{\hbar}\left[t, t^{-1}\right]$ with the product - (4.4) is a proper subalgebra of the deformation quantization algebra $\left(A\left[t, t^{-1}\right][[\hbar]], \cdot\right)$ of $X \times \mathbb{C}^{\times}$. Thus, in order to apply the results of deformation quantization to $A_{\hbar}\left[t, t^{-1}\right]$ we need to impose certain restrictions on the formal power series of polyvector fields and exterior forms on $X \times \mathbb{C}^{\times}$.

We will denote by $T_{\text {poly }}^{\bullet}\left(X \times \mathbb{C}^{\times}\right)[[\hbar]]_{r e s t r}$ the graded Lie algebra of formal power series of polyvector fields on $X \times \mathbb{C}^{\times}$whose componets have bounded powers in $t$. Similarly, $\mathcal{A}^{\bullet}\left(X \times \mathbb{C}^{\times}\right)[[\hbar]]_{\text {restr }}$ will denote the graded $T_{\text {poly }}^{\bullet}\left(X \times \mathbb{C}^{\times}\right)[[\hbar]]_{\text {restr }}$-module of formal power series of exterior forms on $X \times \mathbb{C}^{\times}$whose components have bounded powers in $t$.

The bivector field

$$
\pi^{w}=\pi+t \partial_{t} \wedge w
$$

obviously belongs to $T_{\text {poly }}^{\bullet}\left(X \times \mathbb{C}^{\times}\right)[[\hbar]]_{\text {restr }}$. Furthermore, since $w$ is a Poisson vector field for $\pi$, the bivector (4.6) satisfies the Jacobi identity:

$$
\left[\pi^{w}, \pi^{w}\right]_{S N}=0 .
$$

Hence $\pi^{w}$ equips the graded Lie algebra $T_{\text {poly }}^{\bullet}\left(X \times \mathbb{C}^{\times}\right)[[\hbar]]_{\text {restr }}$ and its module $\mathcal{A}^{\bullet}(X \times$ $\left.\mathbb{C}^{\times}\right)[[\hbar]]_{\text {restr }}$ with differentials $\left[\pi^{w},\right]$ and $\mathcal{L}_{\pi^{w}}$, respectively.

We claim that

Proposition 3 There exists a chain of $\left(L_{\infty^{-}}\right)$quasi-isomorphisms connecting the DGLA module $\left(T_{\text {poly }}^{\bullet}\left(X \times \mathbb{C}^{\times}\right)[[\hbar]]_{\text {restr }}, \mathcal{A}^{\bullet}\left(X \times \mathbb{C}^{\times}\right)[[\hbar]]_{\text {restr }}\right)$ with the differentials $\left[\pi^{w},\right]$ and $\mathcal{L}_{\pi^{w}}$ to the DGLA module $\left(C^{\bullet}\left(A_{\hbar}\left[t, t^{-1}\right]\right), C_{\bullet}\left(A_{\hbar}\left[t, t^{-1}\right]\right)\right)$ of the Hochschild (co) chains of the algebra $A_{\hbar}\left[t, t^{-1}\right]$ with the product (4.4)

\footnotetext{
${ }^{6}$ The authors of 8 work in the category of $C^{\infty}$ real manifolds. For this reason we decided to formulate Theorem 2 without referring to the quantization of H. Bursztyn and S. Waldmann 8 .
} 
Proof. The idea of the proof is to start with the corresponding chain of $\left(L_{\infty^{-}}\right)$quasiisomorphisms for the DGLA module $\left(C^{\bullet}\left(A\left[t, t^{-1}\right][[\hbar]]\right), C \bullet\left(A\left[t, t^{-1}\right][[\hbar]]\right)\right)$ of Hochschild (co)chains of the deformation quantization algebra $A\left[t, t^{-1}\right][[\hbar]]$ and show that this chain can be restricted to a chain between desired DGLA modules. In doing this, a very important role will be played by the Euler field

$$
\mathrm{Eu}=t \frac{\partial}{\partial t}
$$

In particular, we will use the following Fedosov differential on $\Omega \bullet\left(\mathcal{S}\left(X \times \mathbb{C}^{\times}\right)\right)$:

$$
D^{t}=d y_{t}\left(t \frac{\partial}{\partial t}-\frac{\partial}{\partial y_{t}}\right)+D
$$

where $y_{t}$ is an auxiliary formal variable and $D$ is the Fedosov differential (2.7) on $X$.

Replacing $X$ by $X \times \mathbb{C}^{\times}$and $D$ by $D^{t}$ in diagrams (2.10), (2.11), and (2.12) we get the chain of $\left(L_{\infty^{-}}\right)$quasi-isomorphisms of DGLA modules:

$$
\begin{aligned}
& T_{\text {poly }}^{\bullet}\left(X \times \mathbb{C}^{\times}\right) \quad \stackrel{\lambda_{T}^{w}}{\longrightarrow}\left(\Omega^{\bullet}\left(X \times \mathbb{C}^{\times}, \mathcal{T}_{\text {poly }}^{\bullet}\right), D^{t},[,]_{S N}\right) \quad \succ \stackrel{\mathcal{K}}{\rightarrow} \\
& \downarrow_{\text {mod }} \quad \downarrow_{\bmod } \\
& \mathcal{A}^{\bullet}\left(X \times \mathbb{C}^{\times}\right) \quad \stackrel{\lambda_{\mathcal{A}}^{w}}{\longrightarrow} \quad\left(\Omega^{\bullet}\left(X \times \mathbb{C}^{\times}, \mathcal{E}^{\bullet}\right), D^{t}\right) \quad \stackrel{\mathcal{S}}{\prec \prec,} \\
& \stackrel{\mathcal{K}}{\succ} \quad\left(\Omega^{\bullet}\left(C^{\bullet}\left(\mathcal{S}\left(X \times \mathbb{C}^{\times}\right)\right)\right), D^{t}+\partial,[,]_{G}\right) \stackrel{\lambda_{D}^{w}}{\longleftarrow} \quad C^{\bullet}\left(A\left[t, t^{-1}\right]\right) \\
& \downarrow_{\text {mod }} \quad \downarrow_{\text {mod }} \\
& \stackrel{\mathcal{S}}{\prec \prec} \quad\left(\Omega^{\bullet}\left(C \bullet\left(\mathcal{S}\left(X \times \mathbb{C}^{\times}\right)\right)\right), D^{t}+\mathfrak{b}\right) \quad \stackrel{\lambda_{C}^{w}}{\longleftarrow} \quad C \bullet\left(A\left[t, t^{-1}\right]\right),
\end{aligned}
$$

where $\lambda_{T}^{w}, \lambda_{\mathcal{A}}^{w}, \lambda_{D}^{w}$, and $\lambda_{C}^{w}$ are the corresponding versions of $\lambda_{T}, \lambda_{\mathcal{A}}, \lambda_{D}$, and $\lambda_{C}$ in (2.10) and (2.11) for $X \times \mathbb{C}^{\times}$.

Twisting the chain (4.9) by the Maurer-Cartan element $\pi^{w}$ (4.6) we get the following chain of $\left(L_{\infty^{-}}\right)$quasi-isomorphisms of DGLA modules:

$$
\begin{aligned}
& T_{\text {poly }}^{\bullet}\left(X \times \mathbb{C}^{\times}\right)[[\hbar]] \quad \stackrel{\lambda_{T}^{w}}{\longrightarrow}\left(\Omega^{\bullet}\left(X \times \mathbb{C}^{\times}, \mathcal{T}_{\text {poly }}^{\bullet}\right)[[\hbar]], D^{t}+\left[\lambda_{T}^{w}\left(\pi^{w}\right),\right],[,]_{S N}\right) \quad \succ \stackrel{\widetilde{\mathcal{K}}}{\rightarrow} \\
& \downarrow_{\bmod } \quad \downarrow_{\bmod } \\
& \mathcal{A}^{\bullet}\left(X \times \mathbb{C}^{\times}\right)[[\hbar]] \quad \stackrel{\lambda_{\mathcal{A}}^{w}}{\longrightarrow} \quad\left(\Omega^{\bullet}\left(X \times \mathbb{C}^{\times}, \mathcal{E}^{\bullet}\right)[[\hbar]], D^{t}+\mathcal{L}_{\lambda_{T}^{w}\left(\pi^{w}\right)}\right) \quad \leftarrow \stackrel{\widetilde{\mathcal{S}}}{\prec \prec,} \\
& \stackrel{\widetilde{\mathcal{K}}}{\rightarrow} \quad\left(\Omega^{\bullet}\left(C^{\bullet}\left(\mathcal{S}\left(X \times \mathbb{C}^{\times}\right)\right)\right), D^{t}+\partial_{\diamond_{w}},[,]_{G}\right) \stackrel{\stackrel{\lambda_{D}^{w}}{\longleftarrow}}{\longleftarrow} C^{\bullet}\left(A\left[t, t^{-1}\right][[\hbar]], *_{w}\right) \\
& \downarrow_{\text {mod }} \quad \downarrow_{\text {mod }}
\end{aligned}
$$

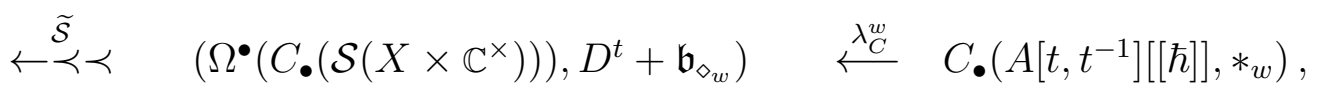

where $T_{\text {poly }}^{\bullet}\left(X \times \mathbb{C}^{\times}\right)[[\hbar]]$ and $\mathcal{A}^{\bullet}\left(X \times \mathbb{C}^{\times}\right)[[\hbar]]$ are considered with the differentials $\left[\pi^{w},\right]_{S N}$ and $\mathcal{L}_{\pi^{w}}$ respectively, $*_{w}$ is a star-product on $A\left[t, t^{-1}\right][[\hbar]]$, and $\diamond_{w}$ is its lift to the algebra $\mathcal{S}\left(X \times \mathbb{C}^{\times}\right)[[\hbar]]:$

$$
\diamond_{w}=\lambda_{D}^{w}\left(*_{w}\right)
$$



by

Let us note that the lift of the Euler field $\mathrm{Eu}(4.7)$ to $\Omega^{0}\left(X \times \mathbb{C}^{\times}, \mathcal{T}_{\text {poly }}^{0}\right) \cap \operatorname{ker} D^{t}$ is given

$$
\lambda_{T}^{w}(\mathrm{Eu})=\partial_{y_{t}}
$$

It is not hard to see that the maps in the chain (4.9) are compatible with the Euler field $\mathrm{Eu}$ (4.7) in the following sense

$$
\begin{aligned}
& \lambda_{T}^{w}\left([\mathrm{Eu}, \gamma]_{S N}\right)=\left[\partial_{y_{t}}, \lambda_{T}^{w}(\gamma)\right]_{S N}, \quad \lambda_{\mathcal{A}}^{w}\left(\mathcal{L}_{\mathrm{Eu}} \eta\right)=\mathcal{L}_{\partial_{y_{t}}} \lambda_{\mathcal{A}}^{w}(\eta) \\
& {\left[\partial_{y_{t}}, \mathcal{K}_{n}\left(\gamma_{1}, \ldots, \gamma_{n}\right)\right]_{G} }=\sum_{j=1}^{n} \mathcal{K}_{n}\left(\gamma_{1}, \ldots, \gamma_{j-1},\left[\partial_{y_{t}}, \gamma_{j}\right]_{S N}, \gamma_{j+1}, \ldots, \gamma_{n}\right) \\
& \mathcal{L}_{\partial_{y_{t}}} \mathcal{S}_{n}\left(\gamma_{1}, \ldots, \gamma_{n} ; c\right)= \sum_{j=1}^{n} \mathcal{S}_{n}\left(\gamma_{1}, \ldots, \gamma_{j-1},\left[\partial_{y_{t}}, \gamma_{j}\right]_{S N}, \gamma_{j+1}, \ldots, \gamma_{n} ; c\right)+ \\
&+\mathcal{S}_{n}\left(\gamma_{1}, \ldots, \gamma_{n} ; R_{\partial_{y_{t}}} c\right) \\
& \lambda_{D}^{w}\left([\mathrm{Eu}, P]_{G}\right)=\left[\partial_{y_{t}}, \lambda_{D}^{w}(P)\right]_{G}, \quad \lambda_{C}^{w}\left(R_{\mathrm{Eu}} b\right)=R_{\partial_{y_{t}}} \lambda_{C}^{w}(b),
\end{aligned}
$$

where $\gamma \in T_{\text {poly }}^{\bullet}\left(X \times \mathbb{C}^{\times}\right), \eta \in \mathcal{A}^{\bullet}\left(X \times \mathbb{C}^{\times}\right), \gamma_{j} \in \Omega^{\bullet}\left(X \times \mathbb{C}^{\times}, \mathcal{T}_{\text {poly }}^{\bullet}\right), c \in \Omega^{\bullet}\left(C \bullet\left(\mathcal{S}\left(X \times \mathbb{C}^{\times}\right)\right)\right)$, $P \in C^{\bullet}\left(A\left[t, t^{-1}\right]\right)$, and $b \in C_{\bullet}\left(A\left[t, t^{-1}\right]\right)$.

On the other hand $\left[\mathrm{Eu}, \pi^{w}\right]_{S N}=0$. Hence the maps in the chain (4.10) are also compatible with the Euler field $\mathrm{Eu}$ and $*_{w}$ can be chosen to satisfy the property

$$
\operatorname{Eu}\left(a_{1} *_{w} a_{2}\right)=\operatorname{Eu}\left(a_{1}\right) *_{w} a_{2}+a_{1} *_{w} \operatorname{Eu}\left(a_{2}\right), \quad a_{1}, a_{2} \in A\left[t, t^{-1}\right][[\hbar]] .
$$

Thus, since the Euler field "counts the powers" in $t$, the chain (4.10) restricts to the chain of $\left(L_{\infty^{-}}\right)$quasi-isomorphisms which connects the DGLA module $\left(T_{\text {poly }}^{\bullet}\left(X \times \mathbb{C}^{\times}\right)[[\hbar]]_{\text {restr }}, \mathcal{A}^{\bullet}(X \times\right.$ $\left.\left.\mathbb{C}^{\times}\right)[[\hbar]]_{\text {restr }}\right)$ with the differentials $\left[\pi^{w},\right]$ and $\mathcal{L}_{\pi^{w}}$ to the DGLA module $\left(C^{\bullet}\left(A[[\hbar]]\left[t, t^{-1}\right]\right)\right.$, $\left.C_{\bullet}\left(A[[\hbar]]\left[t, t^{-1}\right]\right)\right)$ of the Hochschild (co)chains of the algebra $A[[\hbar]]\left[t, t^{-1}\right]$ with the product $*_{w}$.

Our purpose now is to prove that the algebra $A_{\hbar}\left[t, t^{-1}\right]$ with the product (4.4) is isomorphic to the algebra $A[[\hbar]]\left[t, t^{-1}\right]$ with the product $*_{w}$.

It is obvious that

$$
\left.\lambda_{T}^{w}\right|_{A}=\lambda_{T}
$$

Furthermore, $w, t, \pi^{w}$ give us the following $D^{t}$-flat sections of $\mathcal{T}_{\text {poly }}^{\bullet}[[\hbar]]$ on $X \times \mathbb{C}^{\times}$

$$
\lambda_{T}^{w}(w)=\lambda_{T}(w), \quad \lambda_{T}^{w}(t)=t e^{y_{t}},
$$

and

$$
\lambda_{T}^{w}\left(\pi^{w}\right)=\lambda_{T}(\pi)+\partial_{y_{t}} \wedge \lambda_{T}(w)
$$

where, by abuse of notation, we denote by $\lambda_{T}(w)\left(\operatorname{resp} . \lambda_{T}(\pi)\right)$ the natural lift of the fiberwise vector (resp. bivector) on $T X$ to $T\left(X \times \mathbb{C}^{\times}\right)$

Notice that the vector field $w\left(\right.$ on $\left.X \times \mathbb{C}^{\times}\right)$is log-Hamiltonian with respect to $\pi^{w}$ (4.6) . More precisely,

$$
w=-t^{-1}\left[\pi^{w}, t\right]_{S N} .
$$

Therefore, due to Theorem 4 there exists a function $\widetilde{t} \in A\left[t, t^{-1}\right][[\hbar]]$ such that

$$
\widetilde{t}=t \quad \bmod \hbar,
$$


and for every $a \in A\left[t, t^{-1}\right][[\hbar]]$

$$
\exp \left(\mathcal{D}_{w}^{t}\right)(a)=\widetilde{t} *_{w} a *_{w} \tilde{t}^{-1}
$$

where the inverse $\widetilde{t}^{-1}$ is taken in the algebra $\left(A\left[t, t^{-1}\right][[\hbar]], *_{w}\right)$ and $\mathcal{D}_{w}^{t}$ denotes the derivation of this algebra corresponding to the Poisson vector field $w$ on $X \times \mathbb{C}^{\times}$.

The compatibility of the chain of maps (4.10) with the Euler field $\mathrm{Eu}$ (4.7) implies that $\tilde{t}$ as well as its inverse $\widetilde{t}^{-1}$ belongs to the subalgebra $A[[\hbar]]\left[t, t^{-1}\right] \subset A\left[t, t^{-1}\right][[\hbar]]$.

The desired isomorphism $\tau$ from $A_{\hbar}\left[t, t^{-1}\right]$ to $\left(A[[\hbar]]\left[t, t^{-1}\right], *_{w}\right)$ is then defined on generators as follows

$$
\tau(a)=a, \quad \tau(t)=\widetilde{t},
$$

where $a \in A$.

It is Equation (4.18) which implies that (4.20) is indeed an isomorphism of $\mathbb{C}[[\hbar]]$-modules.

To show that (4.20) is a map of algebras we need to check that

$$
a *_{w} b=a * b, \quad \forall \quad a, b \in A[[\hbar]],
$$

and

$$
\widetilde{t} *_{w} a *_{w} \widetilde{t}^{-1}=\exp \left(\mathcal{D}_{w}\right)(a), \quad \forall \quad a \in A[[\hbar]] .
$$

Equation (4.21) follows from (4.14), (4.16) and the identity

$$
\begin{gathered}
\mathcal{K}_{n}\left(\lambda_{T}(\pi)+\partial_{y_{t}} \wedge \lambda_{T}(w), \lambda_{T}(\pi)+\partial_{y_{t}} \wedge \lambda_{T}(w), \ldots, \lambda_{T}(\pi)+\partial_{y_{t}} \wedge \lambda_{T}(w)\right)\left(s_{1}, s_{2}\right)= \\
\mathcal{K}_{n}\left(\lambda_{T}(\pi), \lambda_{T}(\pi), \ldots, \lambda_{T}(\pi)\right)\left(s_{1}, s_{2}\right),
\end{gathered}
$$

where $\mathcal{K}_{n}$ are the structure maps of the quasi-isomorphism $\mathcal{K}$ in (4.9) and $s_{1}, s_{2}$ are sections of $\mathcal{S}\left(X \times \mathbb{C}^{\times}\right)$satisfying the equations

$$
\frac{\partial}{\partial y_{t}} s_{1}=\frac{\partial}{\partial y_{t}} s_{2}=0
$$

To prove (4.22) it suffices to show that

$$
\mathcal{D}_{w}^{t}(a)=\mathcal{D}_{w}(a), \quad \forall \quad a \in A[[\hbar]],
$$

where in the left hand side $w$ is viewed as a vector field on $X \times \mathbb{C}^{\times}$and in the right hand side $w$ is viewed as a vector field on $X$.

Again (4.23) follows from (4.14), (4.15), (4.16) and the identity

$$
\begin{gathered}
\mathcal{K}_{n+1}\left(\lambda_{T}(\pi)+\partial_{y_{t}} \wedge \lambda_{T}(w), \lambda_{T}(\pi)+\partial_{y_{t}} \wedge \lambda_{T}(w), \ldots, \lambda_{T}(\pi)+\partial_{y_{t}} \wedge \lambda_{T}(w), \lambda_{T}(w)\right)(s)= \\
\mathcal{K}_{n+1}\left(\lambda_{T}(\pi), \lambda_{T}(\pi), \ldots, \lambda_{T}(\pi), \lambda_{T}(w)\right)(s),
\end{gathered}
$$

$\mathcal{K}_{n}$ are, as above, the structure maps of the quasi-isomorphism $\mathcal{K}$ in (4.9) and $s$ is a section of $\mathcal{S}\left(X \times \mathbb{C}^{\times}\right)$satisfying the equation

$$
\frac{\partial}{\partial y_{t}} s=0
$$

This concludes the proof of Proposition 3 . 


\section{Criterion of unimodularity}

Let, as above, $X$ be a smooth complex affine variety with trivial canonical bundle. $\operatorname{dim} X=$ $d, \pi_{1}$ is a Poisson structure on $X$, and $A_{\hbar}$ is a deformation quantization algebra of $\pi_{1}$ with Kontsevich's class represented by the formal series $\pi$ (2.20).

We have

Theorem 3 The Van den Bergh dualizing module

$$
U_{A_{\hbar}}=\operatorname{Ext}_{A_{\hbar}^{e}}^{d}\left(A_{\hbar}, A_{\hbar} \otimes A_{\hbar}\right)
$$

of $A_{\hbar}$ is isomorphic to $A_{\hbar}$ as a bimodule if and only if the Poisson structure $\pi$ (2.20) is unimodular.

Although the "if" part of this statement is an immediate corollary of Theorem 2, we give here an independent proof of both implications because we will use Theorem 3 in full generality in the proof of Theorem 2 .

Proof of Theorem 3. Let us prove the implication $\Rightarrow$.

Due to [38] the condition $U_{A_{\hbar}} \cong A_{\hbar}$ implies that we have an isomorphism

$$
V: H H^{0}\left(A_{\hbar}, A_{\hbar}\right) \stackrel{\sim}{\rightarrow} H H_{d}\left(A_{\hbar}, A_{\hbar}\right) .
$$

On the other hand, the formality theorems for Hochschild (co)chains [11], 27], [35] provide us with isomorphisms

$$
\begin{aligned}
& \mu_{1}: H P^{0}(X, \pi) \stackrel{\sim}{\rightarrow} H H^{0}\left(A_{\hbar}, A_{\hbar}\right), \\
& \mu_{2}: H H_{d}\left(A_{\hbar}, A_{\hbar}\right) \stackrel{\sim}{\rightarrow} H P_{d}(X, \pi),
\end{aligned}
$$

where $H P^{\bullet}(X, \pi)$ denotes the Poisson cohomology (2.22), and $H P_{\bullet}(X, \pi)$ denotes the Poisson homology [6], [30]. The latter is, by definition, the homology of the complex $\mathcal{A}^{\bullet}(X)[[\hbar]]$ of exterior forms on $X$ with the differential $\mathcal{L}_{\pi}$ :

$$
H_{\bullet}(X, \pi)=H_{\bullet}\left(\mathcal{A}^{\bullet}(X)[[\hbar]], \mathcal{L}_{\pi}\right) .
$$

Taking the class $[1] \in H P^{0}(X, \pi)$ represented by the function $1 \in A=\mathcal{O}(X)$ we get the class

$$
\mu_{2} \circ V \circ \mu_{1}([1]) \in H P_{d}(X, \pi) .
$$

This class is represented by a formal power series:

$$
\widetilde{\omega}=\omega_{0}+\hbar \omega_{1}+\cdots \in \Gamma\left(\wedge^{d} T^{*} X\right)[[\hbar]]
$$

such that

$$
\mathcal{L}_{\pi} \omega=0 .
$$

Thus, in view of Proposition 1, it suffices to prove that $\omega_{0}$ is nowhere vanishing. For this we consider the Van den Bergh isomorphism (5.2) modulo $\hbar$.

Indeed, modulo $\hbar$ the map (5.2) gives us the Van den Bergh isomorphism

$$
V_{0}: H H^{0}(A, A) \stackrel{\sim}{\rightarrow} H H_{d}(A, A)
$$

for the commutative algebra $A=\mathcal{O}(X)$. 
Due to Hochschild-Kostant-Rosenberg theorem [23] the map $V_{0}$ is an isomorphism from $A$ onto $A$. This observation immediately implies that $\omega_{0}$ is a nowhere vanishing form and the implication $\Rightarrow$ follows.

Let us prove the implication $\Leftarrow$. Since $\pi$ is unimodular Proposition 1 implies that there exists a formal power series (5.6) of top degree forms satisfying (5.7) and starting with a nowhere vanishing form $\omega_{0}$.

Isomorphism (5.4) provides us with a class $\mu_{2}^{-1}([\widetilde{\omega}])$ in $H H_{d}\left(A_{\hbar}, A_{\hbar}\right)$, where $[\widetilde{\omega}]$ is the class of the cycle $\widetilde{\omega}$ in the Poisson chain complex.

On the other hand the Van den Bergh theorem gives us an isomorphism

$$
\widetilde{V}: H H_{d}\left(A_{\hbar}, A_{\hbar}\right) \stackrel{\sim}{\rightarrow} H H^{0}\left(A_{\hbar}, \nu^{-1} A_{\hbar}\right) .
$$

where $\nu$ is the modular automorphism of $A_{\hbar}$.

Let us denote by $b$ a representative of the image $\widetilde{V}\left(\mu_{2}^{-1}([\widetilde{\omega}])\right)$ of the class $\mu_{2}^{-1}([\widetilde{\omega}])$ under the map $\widetilde{V}$. By definition, $b \in A_{\hbar}$, and hence is a formal power series

$$
b=b_{0}+\hbar b_{1}+\hbar^{2} b_{2} \ldots .
$$

Considering the isomorphisms $\mu_{2}$ (5.4) and $\widetilde{V}$ (5.9) modulo $\hbar$ we conclude that $b_{0}$ is invertible in $A$. Hence $b$ is an invertible element of $A_{\hbar}$.

On the other hand the cocycle condition for $b$

$$
\nu^{-1}(a) * b-b * a=0, \quad a \in A_{\hbar}
$$

implies that for every $a \in A_{\hbar}$

$$
\nu^{-1}(a)=b * a * b^{-1},
$$

where $b^{-1}$ is the inverse of $b$ in the algebra $A_{\hbar}$.

Thus, the automorphism $\nu$ is inner and the implication $\Leftarrow$ follows.

\section{Proof of Theorem 2.}

Let us pick on $X$ a nowhere vanishing top degree form

$$
\omega \in \Gamma\left(\wedge^{\operatorname{dim} X} T^{*} X\right)
$$

and assign to this form the modular vector field $v \in \hbar \Gamma(T X)[[\hbar]]$ of the Poisson structure $\pi$ (2.20). It is clear from the definition (3.4) that $v$ is a formal power series of vector fields

$$
v=\hbar v_{1}+\hbar^{2} v_{2}+\ldots
$$

starting with $\hbar v_{1}$ where $v_{1}$ is the modular vector field of the initial Poisson structure $\pi_{1}$ on $X$.

Equation (3.5) implies that $v$ is a cocycle in the Poisson cochain complex of $\pi$. Thus using the construction of subsection 2.1 we can lift $v$ to a derivation

$$
\mathcal{D}_{v}: A_{\hbar} \rightarrow A_{\hbar}
$$

of $A_{\hbar}$ such that

$$
\mathcal{D}_{v}=v \quad \bmod \hbar^{2}
$$

Let us consider the automorphism of $A_{\hbar}$ entering the statement of Theorem 2

$$
\phi=\exp \left(\mathcal{D}_{v}\right)
$$


and define an algebra structure on $A_{\hbar}\left[t, t^{-1}\right]$ by the following rule:

$$
\left(a t^{m}\right) \cdot\left(b t^{k}\right)=a * \phi^{m}(b) t^{m+k},
$$

where $a, b \in A_{\hbar}$ and $m, k$ are arbitrary integers.

Let us now analyze the bimodule structure on

$$
\operatorname{Ext}_{\left(A_{\hbar}\left[t, t^{-1}\right]\right)^{e}}\left(A_{\hbar}\left[t, t^{-1}\right], A_{\hbar}\left[t, t^{-1}\right] \otimes A_{\hbar}\left[t, t^{-1}\right]\right)
$$

using arguments of homological algebra.

For this we extend the action of the inverse

$$
\psi=\phi^{-1}
$$

of the automorphism $\phi(6.4)$ to the Hochschild complex

$$
C^{\bullet}\left(A_{\hbar}, A_{\hbar} \otimes A_{\hbar}\right)
$$

in the natural way:

$$
\psi(P)\left(a_{1}, \ldots, a_{k}\right)=\phi^{-1}\left[P\left(\phi\left(a_{1}\right), \ldots, \phi\left(a_{k}\right)\right)\right]
$$

where

$$
\phi^{-1}\left[b_{1} \otimes b_{2}\right]=\phi^{-1}\left(b_{1}\right) \otimes \phi^{-1}\left(b_{2}\right),
$$

and

$$
P \in C^{k}\left(A_{\hbar}, A_{\hbar} \otimes A_{\hbar}\right) .
$$

The action (6.7) is compatible with the Hochschild coboundary operator and with the internal $A_{\hbar}$-bimodule structure, namely

$$
\psi(P) \cdot \operatorname{int} \phi^{-1}(a)=\psi(P \cdot \text { int } a), \quad \phi^{-1}(a) \cdot \operatorname{int} \psi(P)=\psi(a \cdot \text { int } P) .
$$

Thus, if we fix an isomorphism between the $A_{\hbar^{-} \text {-bimodules }}$

$$
\operatorname{Ext}^{d}\left(A_{\hbar}, A_{\hbar} \otimes A_{\hbar}\right) \cong A_{\hbar} \nu
$$

then the action (6.7) induces the following isomorphism $\hat{\psi}$

$$
\hat{\psi}: A_{\hbar} \nu \rightarrow \phi^{-1} A_{\hbar} \nu \phi^{-1}
$$

from the $A_{\hbar}$-bimodule $A_{\hbar} \nu$ to the $A_{\hbar}$-bimodule $7 \phi^{-1} A_{\hbar} \nu \phi^{-1}$.

The isomorphism $\hat{\psi}$ is uniquely determined by the image of $1 \in A_{\hbar} \nu$. Let us denote this image by $b_{\psi}$ :

$$
b_{\psi}=\hat{\psi}(1) \text {. }
$$

Since $\hat{\psi}$ is an isomorphism the element $b_{\psi}$ has to be invertible in $A_{\hbar}$.

We claim that

Proposition 4 Given the isomorphism (6.9) we may construct the isomorphism of $A_{\hbar}\left[t, t^{-1}\right]$ bimodules

$$
\operatorname{Ext}_{\left(A_{\hbar}\left[t, t^{-1}\right]\right)^{e}}\left(A_{\hbar}\left[t, t^{-1}\right], A_{\hbar}\left[t, t^{-1}\right] \otimes A_{\hbar}\left[t, t^{-1}\right]\right) \cong \begin{cases}A_{\hbar}\left[t, t^{-1}\right] \widetilde{\nu} & \text { if } \bullet=d+1 \\ 0 & \text { otherwise }\end{cases}
$$

\footnotetext{
${ }^{7}$ Hence $\phi \nu \phi^{-1}$ differs from $\nu$ by an inner automorphism.
} 
where

$$
\widetilde{\nu}(a)=\nu(a), \quad \forall a \in A_{\hbar},
$$

and

$$
\widetilde{\nu}(t)=t b_{\psi} .
$$

The isomorphism (6.12) is compatible with the natural action of the Euler field (4.7).

Proof. We remark that $A_{\hbar}\left[t, t^{-1}\right]$ is the twisted group algebra of the group $\mathbb{Z}$. Thus, as a $A_{\hbar}\left[t, t^{-1}\right]$-bimodule, $A_{\hbar}\left[t, t^{-1}\right]$ admits the following free resolution:

$$
C_{\bullet}\left(\mathbb{Z}, C_{\bullet}\left(A_{\hbar}, A_{\hbar}\left[t, t^{-1}\right] \otimes A_{\hbar}\left[t, t^{-1}\right]\right)\right),
$$

where $C_{\bullet}(\mathbb{Z}$,$) denotes the standard chain complex of the group \mathbb{Z}$, the (right) $\mathbb{Z}$-module structure on the chains of the complex $C_{\bullet}\left(A_{\hbar}, A_{\hbar}\left[t, t^{-1}\right] \otimes A_{\hbar}\left[t, t^{-1}\right]\right)$ is given by

$$
\begin{gathered}
\left(a t^{n}, a_{1}, \ldots, a_{k}, b t^{m}\right) t=\left(a t^{n+1}, \phi^{-1}\left(a_{1}\right), \ldots, \phi^{-1}\left(a_{k}\right), \phi^{-1}(b) t^{m-1}\right), \\
a, a_{1}, \ldots, a_{k}, b \in A_{\hbar}
\end{gathered}
$$

and for the definition of the Hochschild chain complex $C_{\bullet}\left(A_{\hbar}, A_{\hbar}\left[t, t^{-1}\right] \otimes A_{\hbar}\left[t, t^{-1}\right]\right)$ we use

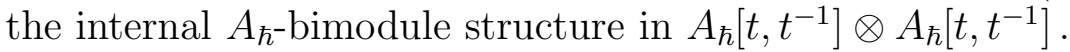

The acyclicity of the resolution (6.15) is proved in the beginning of section 3 in [13] for an arbitrary discrete group acting on an arbitrary associative algebra with unit.

Using this resolution we conclude that the $A_{\hbar}$-bimodules

$$
\operatorname{Ext}_{\left(A_{\hbar}\left[t, t^{-1}\right]\right)^{e}}\left(A_{\hbar}\left[t, t^{-1}\right], A_{\hbar}\left[t, t^{-1}\right] \otimes A_{\hbar}\left[t, t^{-1}\right]\right)
$$

can be computed as the total cohomology of the following double complex

$$
C^{\bullet}\left(\mathbb{Z}, C^{\bullet}\left(A_{\hbar}, A_{\hbar}\left[t, t^{-1}\right] \otimes A_{\hbar}\left[t, t^{-1}\right]\right)\right),
$$

where $C^{\bullet}(\mathbb{Z}, \quad)$ denotes the standard cochain complex of the group $\mathbb{Z}$, the (right) $\mathbb{Z}$-module structure on the cochains of the complex $C^{\bullet}\left(A_{\hbar}, A_{\hbar}\left[t, t^{-1}\right] \otimes A_{\hbar}\left[t, t^{-1}\right]\right)$ is given by

$$
\begin{gathered}
(P T)\left(a_{1}, \ldots, a_{k}\right)=t \cdot{ }_{\text {ext }} P\left(\phi^{-1}\left(a_{1}\right), \ldots, \phi^{-1}\left(a_{k}\right)\right) \cdot \operatorname{ext} t^{-1}, \\
P \in C^{k}\left(A_{\hbar}, A_{\hbar}\left[t, t^{-1}\right] \otimes A_{\hbar}\left[t, t^{-1}\right]\right),
\end{gathered}
$$

where $T$ denotes the (right) action of the generator of $\mathbb{Z}$, ext denotes the multiplication with respect to the external $A_{\hbar}\left[t, t^{-1}\right]$-bimodule structure on $A_{\hbar}\left[t, t^{-1}\right] \otimes A_{\hbar}\left[t, t^{-1}\right]$, and the same external bimodule structure is used in the definition of the Hochschild cochain complex $C^{\bullet}\left(A_{\hbar}, A_{\hbar}\left[t, t^{-1}\right] \otimes A_{\hbar}\left[t, t^{-1}\right]\right)$.

Trivial $\mathbb{Z}$-module $\mathbb{C}$ admits the following simple resolution

$$
0 \rightarrow \mathbb{C}\left[t, t^{-1}\right] \stackrel{\cdot(1-t)}{\longrightarrow} \mathbb{C}\left[t, t^{-1}\right] \stackrel{t=1}{\longrightarrow} \mathbb{C} \rightarrow 0 .
$$

Therefore the cohomology of (6.17) can be computed using the simpler double complex

$$
\begin{array}{ccc}
\stackrel{\partial}{\rightarrow} C^{k}\left(A_{\hbar}, A_{\hbar}\left[t, t^{-1}\right] \otimes A_{\hbar}\left[t, t^{-1}\right]\right) & \stackrel{\partial}{\rightarrow} C^{k+1}\left(A_{\hbar}, A_{\hbar}\left[t, t^{-1}\right] \otimes A_{\hbar}\left[t, t^{-1}\right]\right) & \stackrel{\partial}{\rightarrow} \\
\uparrow 1-T & & \uparrow^{1-T} \\
\stackrel{\partial}{\rightarrow} C^{k}\left(A_{\hbar}, A_{\hbar}\left[t, t^{-1}\right] \otimes A_{\hbar}\left[t, t^{-1}\right]\right) & \stackrel{\partial}{\rightarrow} C^{k+1}\left(A_{\hbar}, A_{\hbar}\left[t, t^{-1}\right] \otimes A_{\hbar}\left[t, t^{-1}\right]\right) \stackrel{\partial}{\rightarrow}
\end{array}
$$

This double-complex is bounded from the left and it has length 2 in the vertical direction. 
Since the Hochschild complex $C^{\bullet}\left(A_{\hbar}, A_{\hbar}\left[t, t^{-1}\right] \otimes A_{\hbar}\left[t, t^{-1}\right]\right)$ splits into the following direct sum

$$
C^{\bullet}\left(A_{\hbar}, A_{\hbar}\left[t, t^{-1}\right] \otimes A_{\hbar}\left[t, t^{-1}\right]\right)=\bigoplus_{n, m \in \mathbb{Z}} C^{\bullet}\left(A_{\hbar}, A_{\hbar} t^{n} \otimes t^{m} A_{\hbar}\right)
$$

Equation (4.1) implies that the rows of the double-complex (6.19) are acyclic beyond the dimension $d$. Using this observation it is not hard to show that

$$
H H^{k}\left(A_{\hbar}\left[t, t^{-1}\right], A_{\hbar}\left[t, t^{-1}\right] \otimes A_{\hbar}\left[t, t^{-1}\right]\right)=0
$$

if $k \neq d+1$ and

$$
\begin{gathered}
H H^{d+1}\left(A_{\hbar}\left[t, t^{-1}\right], A_{\hbar}\left[t, t^{-1}\right] \otimes A_{\hbar}\left[t, t^{-1}\right]\right) \cong \\
H H^{d}\left(A_{\hbar}, A_{\hbar}\left[t, t^{-1}\right] \otimes A_{\hbar}\left[t, t^{-1}\right]\right) /[1-\hat{T}]\left(H H^{d}\left(A_{\hbar}, A_{\hbar}\left[t, t^{-1}\right] \otimes A_{\hbar}\left[t, t^{-1}\right]\right)\right),
\end{gathered}
$$

where $\hat{T}$ is the action on $H H^{\bullet}\left(A_{\hbar}, A_{\hbar}\left[t, t^{-1}\right] \otimes A_{\hbar}\left[t, t^{-1}\right]\right)$ induced by (6.18) .

On the other hand, $H H^{d}\left(A_{\hbar}, A_{\hbar}\left[t, t^{-1}\right] \otimes A_{\hbar}\left[t, t^{-1}\right]\right)$ is a free $\mathbb{Z}$-module generated by $H H^{d}\left(A_{\hbar}, A_{\hbar} \otimes A_{\hbar}\left[t, t^{-1}\right]\right)$ and hence

$$
H H^{d+1}\left(A_{\hbar}\left[t, t^{-1}\right], A_{\hbar}\left[t, t^{-1}\right] \otimes A_{\hbar}\left[t, t^{-1}\right]\right) \cong A_{\hbar}\left[t, t^{-1}\right] \nu
$$

as an $A_{\hbar}$-bimodule and a left module over $A_{\hbar}\left[t, t^{-1}\right]$.

To determine the right module structure over $A_{\hbar}\left[t, t^{-1}\right]$ we remark that for every cochain $P \in C^{k}\left(A_{\hbar}, A_{\hbar} \otimes A_{\hbar}\right)$ viewed as a cochain in $C^{k}\left(A_{\hbar}, A_{\hbar}\left[t, t^{-1}\right] \otimes A_{\hbar}\left[t, t^{-1}\right]\right)$

$$
P \cdot \text { int } t=(t \cdot \text { int } \psi(P)) T \text {. }
$$

Due to (6.20) Equation (6.21) implies that the right $A_{\hbar}\left[t, t^{-1}\right]$-module structure of

$$
H H^{d+1}\left(A_{\hbar}\left[t, t^{-1}\right], A_{\hbar}\left[t, t^{-1}\right] \otimes A_{\hbar}\left[t, t^{-1}\right]\right)
$$

is indeed the one in (41).

It is obvious that the automorphism $\widetilde{\nu}(6.13)$, (6.14) commutes with the action of the Euler field $\mathrm{Eu}$ (4.7). Thus the field Eu acts of the $A_{\hbar}\left[t, t^{-1}\right]$-bimodule $A_{\hbar}\left[t, t^{-1}\right] \widetilde{\nu}$ in the natural way. It is clear from the construction that the isomorphism (6.12) is compatible with this action and this completes the proof of Proposition 4 .

Due to Proposition 3 the DGLA module $\left(C^{\bullet}\left(A_{\hbar}\left[t, t^{-1}\right]\right), C_{\bullet}\left(A_{\hbar}\left[t, t^{-1}\right]\right)\right)$ of Hochschild (co)chains of the algebra $A_{\hbar}\left[t, t^{-1}\right]$ with the product (6.5) is quasi-isomorphic to the DGLA module $\left(T_{\text {poly }}^{\bullet}\left(X \times \mathbb{C}^{\times}\right)[[\hbar]]_{\text {restr }}, \mathcal{A}^{\bullet}\left(X \times \mathbb{C}^{\times}\right)[[\hbar]]_{\text {restr }}\right)$ where the DGLA $T_{\text {poly }}^{\bullet}\left(X \times \mathbb{C}^{\times}\right)[[\hbar]]_{\text {restr }}$ carries the differential $\left[\pi^{t},\right]$, the module $\left.\mathcal{A}^{\bullet}\left(X \times \mathbb{C}^{\times}\right)[[\hbar]]_{\text {restr }}\right)$ carries the differential $\mathcal{L}_{\pi^{t}}$ and the Poisson bivector $\pi^{t}$ is given by the formula

$$
\pi^{t}=\pi+t \partial_{t} \wedge v
$$

It is not hard to see that if $\omega$ is the volume form we picked at the beginning of this section then the form

$$
\omega_{t}=\frac{d t}{t^{2}} \wedge \omega
$$

satisfies the equation

$$
\mathcal{L}_{\pi^{t}} \omega_{t}=0
$$

which implies that the Poisson structure (6.22) on $X \times \mathbb{C}^{\times}$is unimodular. 
Due to Proposition 3 , we may apply Theorem 3 to the algebra $A_{\hbar}\left[t, t^{-1}\right]$ and deduce that

$$
\operatorname{Ext}_{\left(A_{\hbar}\left[t, t^{-1}\right]\right)}^{k}\left(A_{\hbar}\left[t, t^{-1}\right], A_{\hbar}\left[t, t^{-1}\right] \otimes A_{\hbar}\left[t, t^{-1}\right]\right) \cong 0
$$

if $k \neq d+1$ and

$$
\operatorname{Ext}_{\left(A_{\hbar}\left[t, t^{-1}\right]\right) e}^{d+1}\left(A_{\hbar}\left[t, t^{-1}\right], A_{\hbar}\left[t, t^{-1}\right] \otimes A_{\hbar}\left[t, t^{-1}\right]\right) \cong A_{\hbar}\left[t, t^{-1}\right]
$$

as $A_{\hbar}\left[t, t^{-1}\right]$-bimodules.

Let us recapitulate the relevant part of the proof of Theorem 3 keeping track of the action of the Euler field Eu (4.7).

Equation (6.24) implies that the form $\omega_{t}(6.23)$ is a cycle in the complex $\mathcal{A}^{\bullet}(X \times$ $\left.\left.\mathbb{C}^{\times}\right)[[\hbar]]_{\text {restr }}\right)$ with the differential $\mathcal{L}_{\pi^{t}}$.

Due to Proposition 3 the homology of the complex $\left.\mathcal{A}^{\bullet}\left(X \times \mathbb{C}^{\times}\right)[[\hbar]]_{\text {restr }}\right)$ with the differential $\mathcal{L}_{\pi^{t}}$ is isomorphic to the Hochschild homology $H H_{\bullet}\left(A_{\hbar}\left[t, t^{-1}\right], A_{\hbar}\left[t, t^{-1}\right]\right)$ of $A_{\hbar}\left[t, t^{-1}\right]$. Furthermore, this isomorphism is compatible with the action of $\mathrm{Eu}$. Thus we may pull the class represented by $\omega_{t}$ to a class in

$$
c \in H H_{d+1}\left(A_{\hbar}\left[t, t^{-1}\right], A_{\hbar}\left[t, t^{-1}\right]\right) .
$$

Since $\mathcal{L}_{\mathrm{Eu}} \omega_{t}=-\omega_{t}$ we have

$$
\operatorname{Eu}(c)=-c .
$$

Using Van den Bergh duality we pull $c$ to a class

$$
c^{\prime} \in H H^{0}\left(A_{\hbar}\left[t, t^{-1}\right], \widetilde{\nu}^{-1} A_{\hbar}\left[t, t^{-1}\right]\right),
$$

where the automorphism $\widetilde{\nu}$ is defined in (6.13) and (6.14).

Due to Proposition 4 Van den Bergh isomorphism

$$
H H_{d+1}\left(A_{\hbar}\left[t, t^{-1}\right], A_{\hbar}\left[t, t^{-1}\right]\right) \cong H H^{0}\left(A_{\hbar}\left[t, t^{-1}\right], \widetilde{\nu}^{-1} A_{\hbar}\left[t, t^{-1}\right]\right)
$$

is compatible with the action of $\mathrm{Eu}$. Hence,

$$
\operatorname{Eu}\left(c^{\prime}\right)=-c^{\prime}
$$

Thus if $b \in A_{\hbar}\left[t, t^{-1}\right]$ represents the class $c^{\prime}$ then

$$
\operatorname{Eu}(b)=-b .
$$

In other words, the element $b$ is of the form

$$
b=b_{0} t^{-1}, \quad b_{0} \in A_{\hbar} .
$$

The same argument as in the proof of Theorem 3 shows that $b_{0}$ is invertible. While Equation (6.13) and the cocycle condition for $b$

$$
\widetilde{\nu}^{-1}(a) * b-b * a=0, \quad \forall \quad a \in A_{\hbar}
$$

imply that

$$
\nu^{-1}(a)=b_{0} * \phi^{-1}(a) * b_{0}^{-1}, \quad \forall a \in A_{\hbar},
$$

where the inverse $b_{0}^{-1}$ is taken in the algebra $A_{\hbar}$.

Thus the automorphism $\nu$ differs from $\phi(6.4)$ by an inner automorphism and Theorem 2 is proved. 


\section{Concluding remarks}

Following V. Ginzburg [21] the algebra $A$ of functions on the smooth affine variety $X$ with trivial canonical bundle gives us an example of a Calabi-Yau algebra. (See Definition 3.2.3. and Corollary 3.3.2 in [21]). Due to remark 3.2.8 in [21], Theorem 3 implies that the deformation quantization algebra $A_{\hbar}$ is a Calabi-Yau algebra if and only if a representative (2.20) of Kontsevich's class of $A_{\hbar}$ is unimodular 8 . This reformulation of Theorem 3 is similar to the Kontsevich-Soibelman conjecture about Calabi-Yau structures on an $A_{\infty}$ algebra $\mathfrak{A}$ (see conjecture 10.2.8 in [28]). We should mention that structures very similar to the CalabiYau structures [28] were introduced and discussed in [15], [16] for a large class of connected graded algebras.

It should be remarked that the results of G. Felder and B. Shoikhet [19] also show a special role of unimodular Poisson manifolds in deformation quantization. More precisely in [19] the authors prove a statement closely related to the cyclic formality conjecture [36], [37. Using this result they show that Kontsevich's star-product with the harmonic angle function is cyclic. Finally, they also prove a generalization of the Connes-Flato-Sternheimer conjecture [9] on closed star-products in the Poisson case. It would be interesting to find a relationship between the results of [19] and Theorem 3 proved in this paper.

We would like to mention recent preprint [31] by S. Launois and L. Richard in which the authors consider the algebra $B_{\hbar}$ of functions on a uniparametrized quantum affine space and notice that, in this case, the modular class of the corresponding Poisson bracket can be restored as the semiclassical limit of the modular automorphism $\nu$ (4.2) of this algebra $B_{\hbar}$. Theorem 2 shows that, in general, the semiclassical limit of the modular automorphism may not restore the modular class. In particular, it is easy to construct an example in which the semiclassical limit of the modular automorphism vanishes, while the modular class is still non-zero.

Theorem 3 shows that, in the unimodular case, we have the Van den Bergh duality [38] isomorphism:

$$
V: H H^{\bullet}\left(A_{\hbar}, A_{\hbar}\right) \stackrel{\sim}{\rightarrow} H H_{d-\bullet}\left(A_{\hbar}, A_{\hbar}\right),
$$

where $d$ is the dimension of $X$.

On the other hand, due to [27] we have an isomorphism between Hochschild cohomology $H H^{\bullet}\left(A_{\hbar}, A_{\hbar}\right)$ of $A_{\hbar}$ and the Poisson cohomology (2.22) of $\pi$ (2.20). Furthermore, due to [12] and [35] we also have an isomorphism between Hochschild homology $H H_{\bullet}\left(A_{\hbar}, A_{\hbar}\right)$ of $A_{\hbar}$ and the Poisson homology (5.5) of $\pi$ (2.20). This observation poses a question about a relationship between the Van den Bergh duality (7.1) and the duality of P. Xu [40] (see Theorem 4.8 in [40]).

In the general (non-unimodular) case a version of Xu's duality (see, for example, Eq. (10) in [31]) involves the Poisson homology with coefficients in a module over the Poisson algebra. A generalization of this duality statement to Lie algebroids was proposed in [18] and extended further to the framework of module categories in [4]. It would be interesting to investigate a relation of this duality to Van den Bergh duality for the corresponding deformation quantization algebra.

We should also mention paper [5], in which K.A. Brown and J.J. Zhang discuss the Van den Bergh duality for noetherian Hopf algebras. They showed that, in this case, the dualizing module is also determined by an outer automorphism of the algebra. They call this outer automorphism the Nakayama automorphism.

\footnotetext{
${ }^{8}$ It is not hard to see that the property of being unimodular does not depend on the choice of the representative.
} 
A natural generalization of the modular class to the case $Q$-manifolds [33] shows up in the quantization of gauge systems. More precisely, it was shown in [33] that this class happens to be the first obstruction to the existence of a quantum master action in the BV quantization.

Finally, we would like to mention papers [10] and [29] in which the authors consider the hierarchy generated by the modular vector field of a Poisson-Nijenhuis manifold and relate it to a hierarchy of bihamiltonian vector fields. It would be interesting to investigate quantum versions of these hierarchies and their relation to quantization of bihamiltonian systems [25], [26].

\section{Appendix. Poisson, Hamiltonian and log-Hamiltonian vector fields.}

In this section we introduce the notion of log-Hamiltonian vector fields and prove some of their useful properties. In paper [8] these vector fields are called "integral derivations". However, we believe that the name "log-Hamiltonian" is more appropriate for such vector fields. Very similar objects show up in literature in various contexts [1], [14, [22], [34].

Let $X$ be a smooth affine variety and $\pi$ be the Poisson structure (3.1). A vector field $w \in \Gamma(T X)[[\hbar]]$ is called a Poisson vector field of $\pi$ if

$$
[\pi, w]_{S N}=0 .
$$

It is called a Hamiltonian vector field if there exists a function $f \in A[[\hbar]]$ such that

$$
w=[\pi, f]_{S N} .
$$

Finally, we say that $w$ is a log-Hamiltonian vector field of $\pi$ if there exists a unit $f$ of $A[[\hbar]]$ such that

$$
w=f^{-1}[\pi, f]_{S N} .
$$

Notice that, the units of the ring $A[[\hbar]]$ are the formal power series in $\hbar$

$$
f=f_{0}+\hbar f_{1}+\hbar^{2} f_{2}+\ldots, \quad f_{i} \in A
$$

starting with a nowhere vanishing function $f_{0} \in A$.

The Poisson vector fields are degree 0 cocycles and the Hamiltonian vector fields are degree 0 coboundaries in the complex (2.21) . Every log-Hamiltonian vector field is a Poisson vector field.

Let us list some simple properties of the log-Hamiltonian vector fields in the following proposition:

\section{Proposition 5}

1. A linear combination of log-Hamiltonian vector fields with integer coefficients is again a log-Hamiltonian vector field.

2. If $w_{1}$ is a Poisson vector field and $w_{2}$ is a log-Hamiltonian vector field then their Lie bracket $\left[w_{1}, w_{2}\right]$ is a Hamiltonian vector field.

3. If $g \in \hbar A[[\hbar]]$ then the Hamiltonian vector field $w=[\pi, g]_{S N}$ is a log-Hamiltonian vector field.

\section{Proof.}


1. follows from the equations

$$
n f^{-1}[\pi, f]_{S N}=f^{-n}\left[\pi, f^{n}\right]_{S N}, \quad f_{1}^{-1}\left[\pi, f_{1}\right]_{S N}+f_{2}^{-1}\left[\pi, f_{2}\right]_{S N}=\left(f_{1} f_{2}\right)^{-1}\left[\pi, f_{1} f_{2}\right]_{S N},
$$

where $n$ is an integer, $f, f_{1}$, and $f_{2}$ are units of $A[[\hbar]]$.

2. Using the fact that $w_{1}$ is a Poisson vector field it is not hard to prove that

$$
\left[w_{1}, f^{-1}[\pi, f]_{S N}\right]_{S N}=\left[\pi, f^{-1} w_{1}(f)\right]_{S N},
$$

which immediately implies 2 .

3. follows from the obvious equation

$$
e^{-g}\left[\pi, e^{g}\right]_{S N}=[\pi, g]_{S N},
$$

where $e^{ \pm g}$ makes sense because $g \in \hbar A[[\hbar]]$.

This proposition shows that the log-Hamiltonian vector fields form a lattice in the space of Poisson vector fields. The classes of the log-Hamiltonian vector fields in $H P^{1}(X, \pi)$ form a lattice in the center of the Lie algebra $H P^{1}(X, \pi)$.

In subsection 2.1 we construct a map $\mathcal{D}(2.23)$ from the $\mathbb{C}[[\hbar]]$-module of Poisson vector fields to the $\mathbb{C}[[\hbar]]$-module of the derivations of the corresponding deformation quantization algebra $A_{\hbar}$.

If a Poisson vector field $w$ starts with $\hbar$ then so does the corresponding derivation $\mathcal{D}_{w}$ and it makes sense to speak about the automorphism $\exp \left(\mathcal{D}_{w}\right)$. Let us prove that

\section{Theorem 4 If}

$$
w=-f^{-1}[\pi, f]_{S N}
$$

for some unit $f \in A[[\hbar]]$ then there is a function $\tilde{f}$ such that

$$
\widetilde{f}=f \quad \bmod \quad \hbar,
$$

and

$$
\exp \left(\mathcal{D}_{w}\right)(a)=\tilde{f} * a * \tilde{f}^{-1}, \quad \forall \quad a \in A_{\hbar},
$$

where $\tilde{f}^{-1}$ is the inverse of $\tilde{f}$ in the algebra $A_{\hbar}$.

Proof. In the proof we use the construction and notation from subsection 2.1. It is also useful to understand the construction of the maps $\lambda_{T}$ and $\lambda_{D}$ from [12] (see Propositions 13 and 14 in chapter 4 of [12]).

It is obvious that the lift $\lambda_{T}(f)$ satisfies the following property

$$
\lambda_{T}(f)=f(1+g),
$$

where $g$ is a section of $\mathcal{S} X$ satisfying the equation

$$
\left.g\right|_{y^{i}=0}=0 .
$$

Therefore, it makes sense to speak about the section

$$
\widetilde{g}=\ln (1+g),
$$

defined via the Taylor power series of $\ln (1+x)$ around the point $x=0$. 
Equation (7.5) implies that

$$
\lambda_{T}(w)=-\lambda_{T}(f)^{-1}\left[\lambda_{T}(\pi), \lambda_{T}(f)\right]_{S N} .
$$

Furthermore, since $\lambda_{T}(f)=f(1+g)$ and $f$ does not depend on the fiber coordinate $y$

$$
\lambda_{T}(w)=-(1+g)^{-1}\left[\lambda_{T}(\pi),(1+g)\right]_{S N},
$$

or equivalently, in terms of $\widetilde{g}(\underline{7.10)}$

$$
\lambda_{T}(w)=-\left[\lambda_{T}(\pi), \widetilde{g}\right]_{S N} .
$$

Since $\lambda_{T}(f)$ is a flat section with respect to the Fedosov connection (2.7), $D \lambda_{T}(f)=0$, we have

$$
D \widetilde{g}=-f^{-1} d f
$$

where $d$ is the de Rham differential.

Combining (7.12) and (7.13) we get

$$
D \widetilde{g}+\left[\lambda_{T}(\pi), \widetilde{g}\right]_{S N}=-\lambda_{T}(w)-f^{-1} d f,
$$

Hence,

$$
D \widetilde{\mathcal{K}}_{1}(\widetilde{g})+\partial_{\diamond} \widetilde{\mathcal{K}}_{1}(\widetilde{g})=-\widetilde{\mathcal{K}}_{1}\left(\lambda_{T}(w)\right)-\widetilde{\mathcal{K}}_{1}\left(f^{-1} d f\right)
$$

Since $f^{-1} d f$ is independent of the fiber coordinates $y^{i}$ we have

$$
\widetilde{\mathcal{K}}_{1}\left(f^{-1} d f\right)=f^{-1} d f .
$$

Therefore,

$$
D \widetilde{\mathcal{K}}_{1}(\widetilde{g})+\partial_{\diamond} \widetilde{\mathcal{K}}_{1}(\widetilde{g})=-\widetilde{\mathcal{K}}_{1}\left(\lambda_{T}(w)\right)-f^{-1} d f
$$

In subsection 2.1 the cochain $\widetilde{\mathcal{K}}_{1}\left(\lambda_{T}(w)\right)$ was denoted by $W$ and the derivation $\mathcal{D}_{w}$ was defined by Equation (2.33). Using this equation we rewrite (7.16) as

$$
D \widetilde{\mathcal{K}}_{1}(\widetilde{g})+\partial_{\diamond} \widetilde{\mathcal{K}}_{1}(\widetilde{g})=-\lambda_{D}\left(\mathcal{D}_{w}\right)-\left(D+\partial_{\diamond}\right) \Phi\left(W_{1}\right)-f^{-1} d f,
$$

where $W_{1}$ is the component of $W$ of exterior degree 1 .

Since $\widetilde{g}$ is a cochain of $\left(\Omega^{\bullet}\left(\mathcal{T}_{\text {poly }}^{\bullet}\right)[[\hbar]],\left[\lambda_{T}(\pi),\right]\right)$ of degree -1 ,

$$
\widetilde{\mathcal{K}}_{1}(\widetilde{g}) \in \Omega^{0}(\mathcal{S} X)[[\hbar]]
$$

Hence, combing the components of exterior degree 0 and 1 in (7.17), we get

$$
\begin{gathered}
\lambda_{D}\left(\mathcal{D}_{w}\right)=-\partial_{\diamond}\left(\widetilde{\mathcal{K}}_{1}(\widetilde{g})+\Phi\left(W_{1}\right)\right) \\
D\left(\widetilde{\mathcal{K}}_{1}(\widetilde{g})+\Phi\left(W_{1}\right)\right)=-f^{-1} d f
\end{gathered}
$$

Since the series $\pi$ (3.1) belongs to $\hbar T_{\text {poly }}^{1}(X)[[\hbar]]$ and $\left.\widetilde{g}\right|_{y^{i}=0}=0$ due to (7.9)

$$
\left.\widetilde{\mathcal{K}}_{1}(\widetilde{g})\right|_{\hbar=y^{i}=0}=0 .
$$

Furthermore, using the equation defining the homotopy operator $\Phi$ (see Eq. (4.36) in chapter 4 of [12]) we conclude that

$$
\left.\Phi\left(W_{1}\right)\right|_{y^{i}}=0
$$


Thus the element

$$
G=\widetilde{\mathcal{K}}_{1}(\widetilde{g})+\Phi\left(W_{1}\right) \in \Gamma(\mathcal{S} X)[[\hbar]]
$$

satisfies the property

$$
\left.G\right|_{\hbar=y^{i}=0}=0
$$

and hence, the $\diamond$-exponent of $G$

$$
\exp _{\diamond}(G)=1+\sum_{k \geq 1} \frac{1}{k !} \underbrace{G \diamond G \diamond \ldots \diamond G}_{k \text { times }}
$$

makes sense.

Equation (7.18) implies that

$$
\exp \left(\lambda_{D}\left(\mathcal{D}_{w}\right)\right)(b)=\exp _{\diamond}(G) \diamond b \diamond \exp _{\diamond}(-G), \quad \forall \quad b \in \Gamma(\mathcal{S} X)[[\hbar]]
$$

and, since $f$ does not depend on fiber coordinates $y^{i}$,

$$
\exp \left(\lambda_{D}\left(\mathcal{D}_{w}\right)\right)(b)=f \exp _{\diamond}(G) \diamond b \diamond f^{-1} \exp _{\diamond}(-G), \quad \forall \quad b \in \Gamma(\mathcal{S} X)[[\hbar]] .
$$

Let us prove that

$$
D\left(f \exp _{\diamond}(G)\right)=0
$$

Indeed,

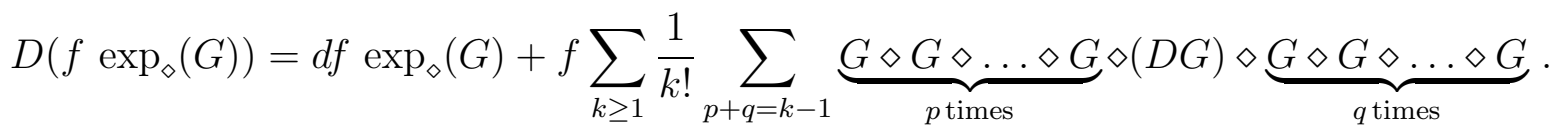

Equation (7.19) implies that $D G=-f^{-1} d f$. Therefore,

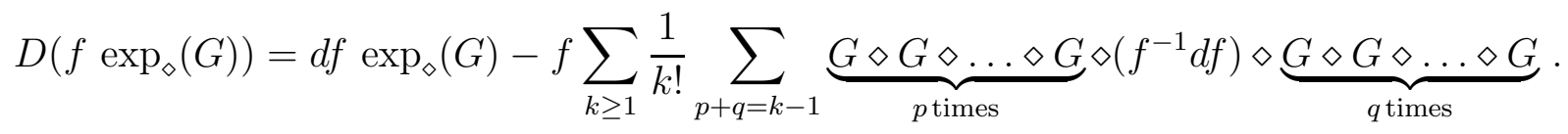

But $f^{-1} d f$ does not depend on the tangent coordinates $y^{i}$, and hence belongs to the center of the algebra $(\Gamma(\mathcal{S} X)[[\hbar]], \diamond)$. Thus, the latter equation gives (7.24).

Equation (7.24) implies that the element $f \exp _{\diamond}(G)$ of $\Omega^{0}\left(C^{-1}(\mathcal{S} X)\right)[[\hbar]]$ belongs to the image of the embedding $\lambda_{D}$ from (2.29). In other words there exists $\tilde{f} \in A[[\hbar]]$ such that

$$
\lambda_{D}(\widetilde{f})=f \exp _{\diamond}(G) .
$$

In fact $\tilde{f}$ can be obtained by simply setting $y^{i}=0$ in $f \exp _{\diamond}(G)$

$$
\tilde{f}=\left.f \exp _{\diamond}(G)\right|_{y^{i}=0}
$$

Using (2.27), (7.23) and (7.25) we immediately conclude that $\tilde{f}$ satisfies equation (7.7). Equation (7.6) follows from the fact that the element $G(7.20)$ satisfies (7.21). This completes the proof of Theorem 4 .

Using claims 2 and 3 of Proposition 5, the Baker-Campbell-Hausdorff formula, and Theorem 4 it is not hard to prove that

Corollary 1 If $w \in \hbar \Gamma(T X)[[\hbar]]$ is Poisson vector field and $w_{\log }$ is a log-Hamiltonian vector field then the automorphism

$$
\exp \left(\mathcal{D}_{w}+\mathcal{D}_{w_{\text {log }}}\right) \circ \exp \left(-\mathcal{D}_{w}\right)
$$

of $A_{\hbar}$ is inner. 


\section{References}

[1] A. Alekseev, A. Malkin, and E. Meinrenken, Lie group valued moment maps, J. Diff. Geom. 48, 3 (1998) 445-495; dg-ga/9707021.

[2] F. Bayen, M. Flato, C. Fronsdal, A. Lichnerowicz, and D. Sternheimer, Deformation theory and quantization. I. Deformations of symplectic structures, Ann. Phys. (N.Y.), 111 (1978) 61;

Deformation theory and quantization. II. Physical applications, Ann. Phys. (N.Y.), 110 (1978) 111.

[3] F.A. Berezin, Quantization, Izv. Akad. Nauk. 38 (1974) 1116-1175;

General concept of quantization, Commun. Math. Phys. 40 (1975) 153-174.

[4] J. Block, Duality and equivalence of module categories in noncommutative geometry I, math.QA/0509284

[5] K. A. Brown and J. J. Zhang, Dualising complexes and twisted Hochschild (co)homology for noetherian Hopf algebras, math.RA/0603732.

[6] J.-L. Brylinski, A differential complex for Poisson manifolds, J. Diff. Geom. 28, 1 (1988) $93-114$.

[7] J.-L. Brylinski and G. Zuckerman, The outer derivation of a complex Poisson manifold, J. Reine Angew. Math. 506 (1999) 181-189.

[8] H. Bursztyn and S. Waldmann, Bimodule deformations, Picard groups and contravariant connections, $K$-Theory 31, 1 (2004) 1-37; arXiv:math/0207255.

[9] A. Connes, M. Flato, and D. Sternheimer, Closed star-product and cyclic cohomology, Lett. Math. Phys. 24, 1 (1992) 1-12.

[10] P. A. Damianou and R. L. Fernandes, Integrable hierarchies and the modular class, math.DG/0607784.

[11] V.A. Dolgushev, A Formality theorem for Hochschild chains, Adv. Math. 200, 1 (2006) 51-101; math.QA/0402248

[12] V.A. Dolgushev, A Proof of Tsygan's formality conjecture for an arbitrary smooth manifold, PhD thesis, MIT; math.QA/0504420.

[13] V.A. Dolgushev and P.I. Etingof, Hochschild cohomology of quantized symplectic orbifolds and the Chen-Ruan cohomology, Int. Math. Res. Not. 27 (2005) 1657-1688; math.QA/0410562.

[14] D. Donin and B. Khesin, Pseudodifferential symbols on Riemann surfaces and KricheverNovikov algebras, Commun. Math. Phys. 272, 2 (2007) 507-527.

[15] M. Dubois-Violette, Multilinear forms and graded algebras, math.QA/0604279

[16] M. Dubois-Violette, Graded algebras and multilinear forms, C. R. Acad. Sci. Paris, Ser. I, 341, (2005) 719-724.

[17] Pavel Etingof and Alexei Oblomkov, Quantization, orbifold cohomology, and Cherednik algebras, In Jack, Hall-Littlewood and Macdonald polynomials, 171-182, Contemp. Math., 417, Amer. Math. Soc., Providence, RI, 2006; math.QA/0311005. 
[18] S. Evens, J.-H. Lu, and A. Weinstein, Transverse measures, the modular class and a cohomology pairing for Lie algebroids, Quart. J. Math. Oxford Ser. 2, 50, 200 (1999) 417-436; dg-ga/9610008.

[19] G. Felder and B. Shoikhet, Deformation quantization with traces, Lett. Math. Phys. 53, 1 (2000) 75-86; math.QA/0002057.

[20] M. Gerstenhaber, The cohomology structure of an associative ring, Ann. Math., 78 (1963) 267-288.

[21] V. Ginzburg, Calabi-Yau algebras, math.AG/0612139.

[22] R. Goto, Rozansky-Witten invariants of log-symplectic manifolds, Integrable systems, topology, and physics (Tokyo, 2000), 69-84, Contemp. Math., 309, Amer. Math. Soc., Providence, RI, 2002.

[23] G. Hochschild, B. Kostant, and A. Rosenberg, Differential forms on regular affine algebras, Trans. Amer. Math. Soc. 102 (1962) 383-408.

[24] J. Huebschmann, Duality for Lie-Rinehart algebras and the modular class, J. Reine Angew. Math. 510 (1999) 103-159; dg-ga/9702008.

[25] D.J. Kaup and P.J. Olver, Quantization of biHamiltonian systems, J. Math. Phys. 31 (1990) 113-117.

[26] B. Khesin, A. Levin, and M. Olshanetsky, Bihamiltonian structures and quadratic algebras in hydrodynamics and on non-commutative torus, Commun. Math. Phys. 250 (2004) 581-612; nlin.SI/0309017.

[27] M. Kontsevich, Deformation quantization of Poisson manifolds, Lett. Math. Phys. 66 (2003) 157-216; q-alg/9709040.

[28] M. Kontsevich and Y. Soibelman, Notes on A-infinity algebras, A-infinity categories and non-commutative geometry. I, math.RA/0606241.

[29] Y. Kosmann-Schwarzbach and F. Magri, On the modular classes of Poisson-Nijenhuis manifolds, math.SG/0611202.

[30] J.L. Koszul, Crochet de Schouten-Nijenhuis et cohomologie, Astérisque (1985) Numero Hors Serie, 257-271.

[31] S. Launois and L. Richard, Twisted Poincaré duality for some quadratic Poisson algebras, Lett. Math. Phys. 79, 2 (2007) 161-174.

[32] A. Lichnerowicz, Les variétés de Poisson et leurs algèbres de Lie associées, J. Diff. Geom., 12, 2 (1977) 253-300.

[33] S.L. Lyakhovich and A.A.Sharapov, Characteristic classes of gauge systems, Nucl. Phys. B703 (2004)419-453; hep-th/0407113.

[34] A.V. Odesskii and V.N. Rubtsov, Polynomial Poisson algebras with a regular structure of symplectic leaves, (Russian) Teoret. Mat. Fiz. 133, 1 (2002) 3-23; translation in Theoret. and Math. Phys. 133, 1 (2002) 1321-1337; math.QA/0110032.

[35] B. Shoikhet, A proof of the Tsygan formality conjecture for chains, Adv. Math. 179, 1 (2003) 7 - 37; math.QA/0010321. 
[36] D. Tamarkin and B. Tsygan, Cyclic formality and index theorems. Talk given at the Moshé Flato Conference (2000), Lett. Math. Phys. 56, 2 (2001) 85-97.

[37] B. Tsygan, Formality conjectures for chains, Differential topology, infinite-dimensional Lie algebras, and applications. 261-274, Amer. Math. Soc. Transl. Ser. 2, 194, AMS, Providence, RI, 1999.

[38] M. Van Den Bergh, A Relation between Hochschild Homology and Cohomology for Gorenstein Rings, Proc. Amer. Math. Soc. 126, 5 (1998) 1345-1348;

Erratum to "A Relation between Hochschild Homology and Cohomology for Gorenstein Rings", Proc. Amer. Math. Soc. 130, 9 (2002) 2809-2810.

[39] A. Weinstein, The modular automorphism group of a Poisson manifold, J. Geom. Phys. 23, 3-4 (1997) 379-394.

[40] P. Xu, Gerstenhaber algebras and BV-algebras in Poisson geometry, Commun. Math. Phys. 200, 3 (1999) 545-560; dg-ga/9703001.

Department of Mathematics, Northwestern University, Evanston, IL 60208

E-mail address: vald@math.northwestern.edu 